\title{
From Energy Gradient and Natural Selection to Biodiversity and Stability of Ecosystems
}

\author{
Bo Deng*
}

Department of Mathematics, University of Nebraska-Lincoln, Lincoln, NE 68588, USA

\begin{abstract}
The purpose of this paper is to incorporate well-established ecological principles into a foodweb model consisting of four trophic levels --- abiotic resources, plants, herbivores, and carnivores. The underlining principles include Kimura's neutral theory of genetic evolution, Liebig's Law of the Minimum for plant growth, Holling's functionals for herbivore foraging and carnivore predation, the One-Life Rule for all organisms, and Lotka-Volterra's model for intraand interspecific competitions. Numerical simulations of the model led to the following statistical findings: (a) particular foodwebs can give contradicting observations on biodiversity and productivity, in particular, all known functional forms -- positive, negative, sigmoidal, and unimodal correlations are present in the model; (b) drifting stable equilibria should be expected for ecosystems regardless of their size; (c) resource abundance and specific competitions are the main determining factors for biodiversity, with intraspecific competition enhancing diversity while interspecific competition impeding diversity; (d) endangered species are expected always and loss in lower trophic endangered species are expected at trophication, i.e. the establishment of a higher trophic level of a community. These findings may shed lights on some ongoing debates on biodiversity. In particular, finding (a) implies that the diversity $v s$. ecosystems functioning debate is most likely the result of incompatible particular observations each cannot be generalized. In particular, general causality should not be expected between diversity and productivity. Finding (b) does not support May's theory that large ecosystems are inherently unstable nor Eton's theory that stability requires diversity. However, it lends a strong support to the energetic theory for the latitudinal diversity gradient. Finding (c) supports Darwin's observation on the effect of interspecific competition on diversity. Finding (d) implies that loss of diversity is inevitable with the appearance of a super species like the human race. Our method and result also suggest that although the evolution of particular species cannot be predicted, some general statistic patterns appear to persist. In addition to the aforementioned findings, these persisting patterns include: the trophic succession, the trophic biomass separation in orders of magnitude, the upper bounds in biodiversity in relationship to the intensities of specific competitions despite the enormous possible number of species allowed by genetic mutations.
\end{abstract}

Keywords: Foodweb, chemostat-population model, biodiversity, species richness, ecological stability, ecosystems functioning, intraspecific competition, interspecific competition, Liebig's Law of the Minimum, Holling's disc function, One-Life Rule, competitive species, endangered species, drifting equilibrium, mutation field, succession, trophication.

To those who look at climate and the physical conditions of life as the all-important elements of distribution, these facts ought to cause surprise, as climate and height or depth graduate away insensibly. But when we bear in mind that almost every species, even in its metropolis, would increase immensely in numbers, were it not for other competing species ....

--- Charles Darwin, On the Origin of the Species, 1859

\section{INTRODUCTION}

The latitudinal gradient in species distribution is one of the oldest patterns in biogeography (Darwin 1859, Wallace 1876, Wallace 1878, Darlington 1957, Hawkins 2001). It is near universal with only a few exceptions (Hillebrand 2003). Although there are many theories (Whittaker 1967, Broham 2003, Scheiner 2005, Qian 2007, Bridle 2009), the correlation between the distribution and the climate along the gradient at the global scale is shown to be the strongest (Currie 1991, Currie 2004). Palaeotological studies also suggest that gradual climate changes rather than exogenous catastrophes might have caused some mass extinctions for

*Address correspondence to this author at the Department of Mathematics, University of Nebraska-Lincoln, Lincoln, NE 68588, USA; Tel: 1-402-472-

7219; Fax: 1-402-472-8466; E-mail: bdeng@math.unl.edu

Handling Editor: Helen Regan marine species (Stanley 1984) and the relatively smaller scale extinction through the Cretaceous-Tertiary boundary (Briggs 1995). Hence, the climate or the water-energy theory seems to be the primary component for a unified theory for biodiverstiy whatever it may eventually be (Stanley 1984, Hawkins 2001, Currie 1991, O'Brien 1998, Currie 2004). At smaller scales and for particular systems, there is little consensus on the relationship between biodiversity and productivity (King 1983, Rosenzweig 1995, Doak 1998, Waide 1999, Huston 2000, Kinzig 2001, Dijkstra 2005, Scheiner 2005, Bradley 2009). One school of researchers argue that species-richness is the determining factor for ecosystems functioning (Tilman 1988, McGrady-Steed 1997, Lehman 2000, Kinzig 2001, Tilman 2006) while many others are unconvinced (Schulze 1984, McNaughton 1994, Waide 1999, Huston 2000, Bradley 2009). There are four functional forms for the diversity-productivity relationship -- the positive and negative correlations, the unimodal and 
sigmoidal relations, and they are all present in significant proportions in the literature (Waide 1999). There are no less than 20 descriptive theories for the diverging observations (Scheiner 2005).

By most accounts on another aspect of biodiversity, ecosystems in general are not only diverse but also stable, which led to the causality question between the two. One theory asserts only complex systems can be stable (Elton 1958, Hutchison 1961, Kinzig 2001, Clark 2007) while another theory insists exactly the opposite that complexity is inherently unstable (May 1972, May 1973, Pimm 1984, Pfisterer 2002, May 2009), but both find a common ground in the theory of competition exclusion principle (MacArthur 1964, Armstrong 1980) based on the RosenzweigMacArthur producer-consumer model (Rosenzweig 1963). Yet, both theories are inconsistent with the Lotka-Volterra model (Lotka 1925, Volterra 1926) for competing species which can be stable regardless of its size (MacArthur 1967, Freedman 1985, Ahmad 1996, Montes de Oca 1995, Rozdilsky 2001), a mathematical proof of which was found in the mid-90s (Ahmad 1996, Montes de Oca 1995) but remains largely unknown to ecologists.

From biogeography to theoretical ecology, biodiversity is inextricably linked to all important facets of living systems. Many mathematical models have been proposed but most are used to advance some particular theories, from phenomenologically fitted models (MacArthur 1963, Hillebrand 2003, Doak 1998) to discrete models (Hughes 2000, Ives 2000, McPeek 2008) which violate the Time Invariance Principle for reproducibility of experiments (Deng 2008, Deng 2009) to higher dimensional extensions of the RosenzweigMacArthur model (Lehman 2000, Shurin 2001, Mouquet 2002, Thebault 2005, Gallet 2007, Gross 2007, Goudard 2008) which violate the One-Life Rule (Deng 2008, Deng 2009) for all organisms but support the complexity-instabi- lity theory, and to multi-trophic extensions of the LotkaVolterra model (De Angelis 1975a, Michalski 1999, Allesina 2008) which support the stability-usually theory.

Because of the diverging findings on biodiversity, descriptive modeling seems to have reached its limit to produce a unified narrative due to the unavoidable linguistic ambiguities on which descriptive modeling is based. It seems that only mathematics can provide a concise, quantitative, and mechanistic alternative, as it has done for other branches of physical science. However, using ad hoc, phenomenological, curve-fitting, and non-mechanistic mathematics for particular theories has the tendency to harden and widen the existing divergences of observations, quantitative or descriptive. Thus the purpose of this paper is to consider a mechanistic model based only on well-established biophysical principles, not preconceived to test any particular theory but to establish a set of basal properties which other theories can use as their "null hypotheses" to test against. As we will demonstrate below that these "basal properties" happen to support the followings: the climate theory for the latitudinal species gradient, the stable-usually theory for the complexity-stability problem, all the diversity-productivity functional forms for particular systems, and to support Darwin's competitiondiversity hypothesis.

\section{METHOD}

The model considered for this paper is a chemostattrophic model for foodwebs (Waltman 1983, Smith 1994) with one resource level and three trophic levels. The model's constituent parts are not only well-known but also considered mechanistically sound. It consists of $n_{0}$ abiotic resources $R_{\mathrm{i}}, n_{1}$ plants $P_{\mathrm{i}}, n_{2}$ herbivores $H_{\mathrm{k}}$, and $n_{3}$ carnivores $C_{\mathrm{l}}$, see Fig. (1a) for a schematic representation. It is a system of differential equations given as follows,

$$
\begin{aligned}
\frac{d R_{i}}{d t}= & r_{i}-w_{i} R_{i}-\sum_{j=1}^{n_{1}} \frac{a_{i j}^{(1)} R_{i}}{1+h_{i j}^{(1)} a_{i j}^{(1)} R_{i}} P_{j} \\
& f o r i=1,2, \ldots, n_{0}, \\
\frac{d P_{i}}{d t}= & P_{i}\left[\min _{1 \leq j \leq n_{0}}\left\{b_{j i}^{(1)} \frac{a_{j i}^{(1)} R_{j}}{1+h_{j i}^{(1)} a_{j i}^{(1)} R_{j}}\right\}-d_{i}^{(1)}-m_{i}^{(1)} P_{i}-\sum_{j \neq i} c_{i j}^{(1)} P_{j}-\sum_{j=1}^{n_{2}} \frac{a_{i j}^{(2)}}{1+\sum_{\ell=1}^{n_{1}} h_{\ell j}^{(2)} a_{\ell j}^{(2)} P_{\ell}} H_{j}\right] \\
& \text { for } i=1,2, \ldots, n_{1}, \\
\frac{d H_{i}}{d t}= & H_{i}\left[\sum_{j=1}^{n_{1}} b_{j i}^{(2)} \frac{a_{j i}^{(2)} P_{j}}{1+\sum_{\ell=1}^{n_{1}} h_{\ell i}^{(2)} a_{\ell i}^{(2)} P_{\ell}}-d_{i}^{(2)}-m_{i}^{(2)} H_{i}-\sum_{j \neq i} c_{i j}^{(2)} H_{j}-\sum_{j=1}^{n_{3}} \frac{a_{i j}^{(3)}}{1+\sum_{\ell=1}^{n_{2}} h_{\ell j}^{(3)} a_{\ell j}^{(3)} H_{\ell}} C_{j}\right] \\
& \text { for } i=1,2, \ldots, n_{2}, \\
\frac{d C_{i}}{d t}= & C_{i}\left[\sum_{j=1}^{n_{2}} b_{j i}^{(3)} \frac{a_{j i}^{(3)} H_{j}}{1+\sum_{\ell=1}^{n_{2}} h_{\ell i}^{(3)} a_{\ell i}^{(3)} H_{\ell}}-d_{i}^{(3)}-m_{i}^{(3)} C_{i}-\sum_{j \neq i} c_{i j}^{(3)} C_{j}\right] \\
& \text { for } i=1,2, \ldots, n_{3} .
\end{aligned}
$$




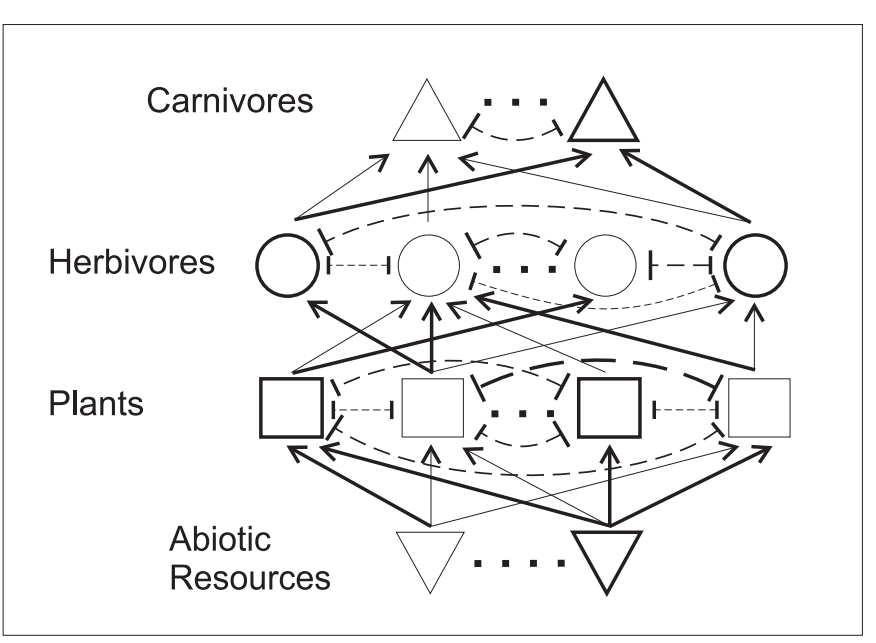

(a)

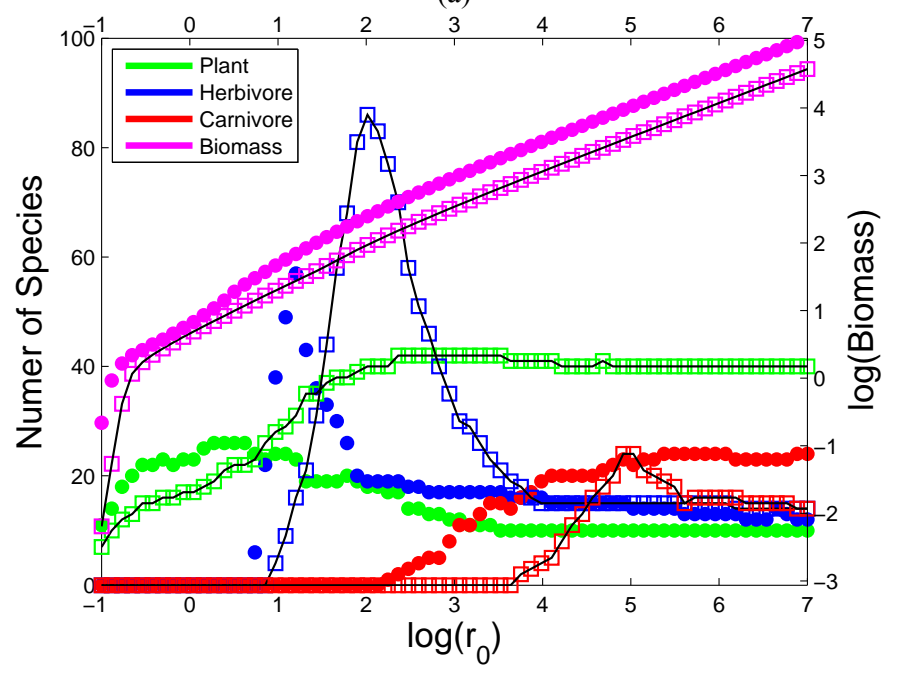

(c)
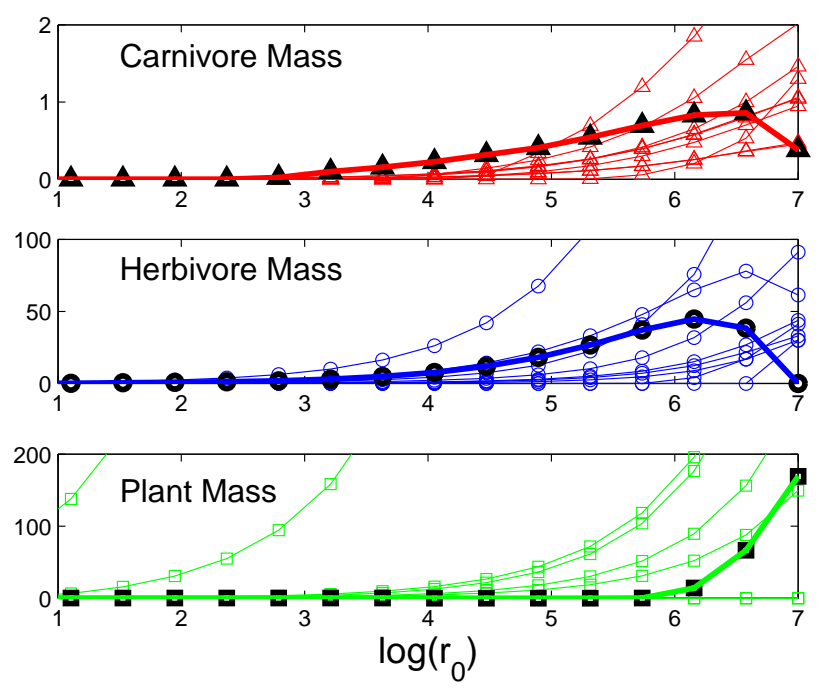

$\operatorname{3in}(\mathrm{b})$

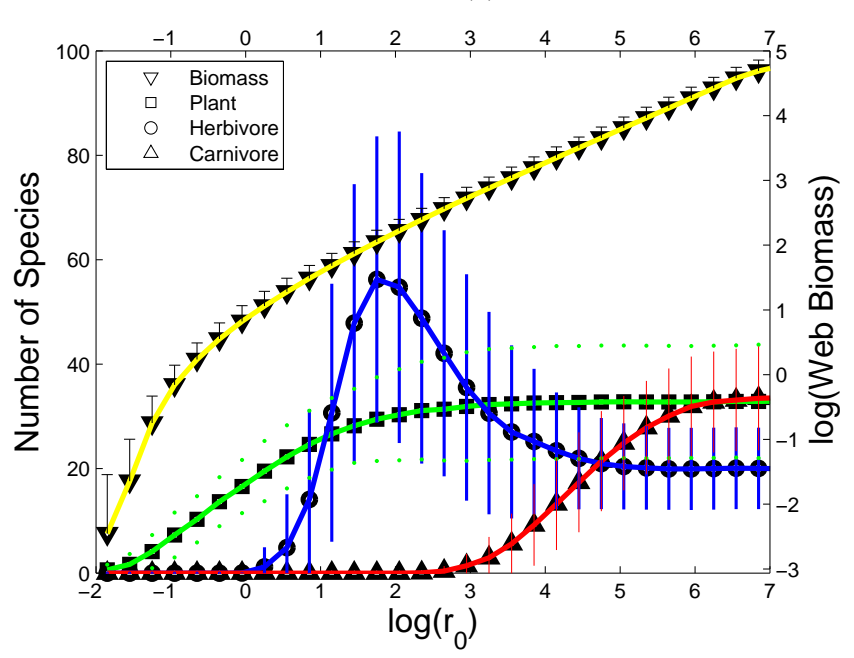

3 in (d)

Fig. (1). (a) A schematic illustration of a food web. Arrows denote donor-recipient relationship between trophics, T-lines denote intraspecific and interspecific competitions. Thicker arrows and T-lines denote greater interactions in strength, and thicker symbols denote species of greater biomass. All connectivity parameters are randomly assigned for the mathematical model. (b) One particular run for Eq. (1), showing the dimensionless biomass of 10 most abundant species in each trophic level. (c) Two particular runs, $\bullet$, $\square$, showing that only the total biomass of each run behaves qualitatively the same. (d) Statistics on means and standard deviations for species numbers against the resource gradient. Each data point was generated from over 100 runs.

The definitions for the variables and parameters are given in Table 1.

Abiotic resources may include essential elements such as light, water, carbon, nitrogen, etc. According to Tilman (1988) there are about 30 elements and molecules required by plants, but there is no limit on $n_{0}$ for the model. One can also consider space as an essential resource for plants (Tilman 1988) and take one of the variables $R_{\mathrm{i}}$ for space. For the abiotic species $R_{\mathrm{i}}, r_{\mathrm{i}}$ is the input/regenerating rate, $w_{\mathrm{i}}$ is the depletion/washout coefficient so that without any uptake by plants $\left(a_{0}^{(1)}=0\right)$ each resource eventually settles down to a steady state equilibrium $R_{i}=r_{i} / w_{i}$ (Waltman 1983, Smith 1994). For example, the equilibrium for space is the available space for plants in a given habitat, that for the light is the amount of solar radiation that the habitat is capable of retaining for plant growth. Similar interpretations apply to water, and other essential chemical elements. To run one simulation of the model, parameters $r_{\mathrm{i}}$ are drown from a uniformly distributed random numbers from interval $\left[0, r_{0}\right]$ with $r_{0}$ defining the range of the distribution and the interval simulating the so-called resource gradient. We will use $r_{0}$ as a bifurcation parameter for most simulations, which can be thought as a measure of resource abundance for a given type of habitats. To simulate the effect of resource limitation in some runs, we will limit some resources to a fixed range but let the range of others vary. The effect of the latter can be thought to correlate, e.g., the PET (Potential Evapotranspiration) measurement of a habitat (Currie 1991, Currie 2004). For each run we will select parameters $w_{\mathrm{i}}$, as well as all other 
parameters the same way as we do for $r_{\mathrm{i}}$, which are summarized in Table 1. More specifically, the set of parameters $r_{\mathrm{i}}, w_{\mathrm{i}}$ for all $1 \leq i \leq n_{0}$ is thought to characterize a particular habitat with uniform means $r_{0} / 2, w_{0} / 2$ respectively. We note that each simulation run with a uniformly small initial population for all species can be thought to simulate the speciation of a geographically isolated habitat or the primary succession or invasion by species of such a habitat, and statistics obtained from an ensemble of such individual simulations can be either thought to characterize some spacial aggregated properties of speciation and/or succession contemporaneously over isolated habitats or temporal aggregated properties of one habitat over a series of speciation and/or succession.

Table 1. Model Variables and Parameters

\begin{tabular}{|c|c|}
\hline$R=\left(R_{1}, R_{2}, \ldots, R_{n_{0}}\right)^{\prime}$ & $n_{0}$ resources \\
\hline$P=\left(P_{1}, P_{2}, \ldots, P_{n_{1}}\right)^{\prime}$ & $n_{1}$ plants \\
\hline$H=\left(H_{1}, H_{2}, \ldots, H_{n_{2}}\right)^{\prime}$ & $n_{2}$ herbivores \\
\hline$C=\left(C_{1}, C_{2}, \ldots, C_{n_{3}}\right)^{\prime}$ & $n_{3}$ carnivores \\
\hline$r=\left[r_{i}\right]=r_{0} *$ rand $\left(n_{0}, 1\right)$ & resource input rate \\
\hline$w=\left[w_{i}\right]=w_{0} *$ rand $\left(n_{0}, 1\right)$ & resource depletion coefficient rate \\
\hline$q^{(k)}=\left[q_{i j}^{(k)}\right]=\left(\operatorname{rand}\left(n_{k-1}, n_{k}\right) \leq q_{0}^{(k)}\right)$ & $\begin{array}{l}\text { trophic-connectivity with } \\
\text { probability } q_{0}^{(k)} \text { with } k \geq 2 \text { and } \\
\qquad q_{i j}^{(1)}=1 \text { for all } i, j\end{array}$ \\
\hline$a^{(k)}=\left[a_{i j}^{(k)}\right]=a_{0}^{(k)} * \operatorname{rand}\left(n_{k-1}, n_{k}\right) * q^{(k)}$ & contact or discovery rate \\
\hline$h^{(k)}=\left[h_{i j}^{(k)}\right]=h_{0}^{(k)} * \operatorname{rand}\left(n_{k-1}, n_{k}\right)$ & uptaking or handling time \\
\hline$b^{(k)}=\left[b_{i j}^{(k)}\right]=b_{0}^{(k)} * \operatorname{rand}\left(n_{k-1}, n_{k}\right)$ & birth-to-consumption ratio \\
\hline$d^{(k)}=\left[d_{i}^{(k)}\right]=d_{0}^{(k)} * \operatorname{rand}\left(n_{k}, 1\right)$ & per-capita death rate \\
\hline$m^{(k)}=\left[m_{i}^{(k)}\right]=m_{0}^{(k)} * \operatorname{rand}\left(n_{k}, 1\right)$ & $\begin{array}{l}\text { intraspecific competition } \\
\text { parameter rate }\end{array}$ \\
\hline$p^{(k)}=\left[p_{i j}^{(k)}\right]=\left(\operatorname{rand}\left(n_{k}, n_{k}\right) \leq p_{0}^{(k)}\right) * v$ & $\begin{array}{c}\text { inter-connectivity with } \\
\text { probability } p_{0}^{(k)} \text { and } v=\left[v_{i j}\right] \\
v_{i i}=0, v_{i j}=1 \text { for } 1 \leq i, j \leq n_{k}\end{array}$ \\
\hline$c^{(k)}=\left[c_{i j}^{(k)}\right]=c_{0}^{(k)} * \operatorname{rand}\left(n_{k}, n_{k}\right) * p^{(k)}$ & $\begin{array}{l}\text { interspecific competition } \\
\text { parameter rate with } c_{i j}=0 \text { for all } i\end{array}$ \\
\hline
\end{tabular}

$\operatorname{rand}(m, n) \quad---$ Matlab function for $m \times n$ matrix of entries uniformly distributed in $[0,1]$.

$(x \leq y)$--- Matlab binary function with $(x \leq y)=1$ or 0 if $x \leq y$ or $x>y$ respectively.

$u_{0}^{(k)}=\max \left\{\left|u_{i j}^{(k)}\right|: 1 \leq i \leq m, 1 \leq j \leq n\right\}--$ the norm or range of a $m \times n$ matrix $u^{(k)}$

To model plant growth, we use the Michaelis-Menten functional form to model plant's resource uptake in rate and Liebig's Law of Minimum to model plant's resource-tobiomass conversion. The Michaelis-Menten form (Michaelis 1913), $a_{i j}^{(1)} R_{i} P_{j} /\left(1+h_{i j}^{(1)} a_{i j}^{(1)} R_{i}\right)$, also known as the MonodJacob form (Monod 1961) and Holling's Type II disc function (Holling 1959), is for rates of irreversible reactions. We use it according to Holling's convention by which $a_{i j}^{(1)}$ is the encounter rate of resource $R_{i}$ by plant $P_{i}$, and $h_{i j}^{(1)}$ is the handling time to assimilate one unit of the resource for growth. The process of resource uptake by plant is assumed to be parallel among all resources, and hence the individual Holling's Type II forms. Since a plant is a package of essential elements in some stoichiometric ratios against each other, such as the water to dry mass ratio, the carbon to nitrogen ratio, or the degree-day to production ratio, etc., the conversion of resources to biomass is assumed to follow Liebig's Law of Minimum with the conversion ratios $b_{j i}^{(1)}$ (Tilman 1988, Deng 2007b, Bradley 2009).

The resource uptake terms modeling the competition for resources by individual plant species without intraspecific nor interspecific interferences. These interferences are modeled by the intraspecific competition term $m_{i}^{(1)} P_{i}$ and the interspecific competition terms $\sum_{i \neq j} c_{i j}^{(1)} P_{j}$. Competition for space should correlate inversely to the coefficient parameters, especially the interspecific parameters $c_{i j}^{(1)}$. Interspecific competition may also include, for example, competition for pollinators. One way to justify these terms is to start with an uptake functional form with interferences $a x /(1+a h x+\mu y+\sigma z)$ with $x$ being a resource, $y$ being a plant, and $z$ being other competing plants (Murdoch 1973, Lawton 1974, Beddington 1975, De Angelis 1975b). The first degree Taylor expansion with respect to $y, z$ gives rise to the intraspecific and interspecific competition terms since $a x /(1+a h x+\mu y+\sigma z) \sim a x /(1+a h x)-m y-c z$. While not all $c_{i j}^{(1)} \geq 0$ must be strictly positive depending on if there is an interspecific competition from plant $P_{j}$ to $P_{i}$, the intraspecific competition parameters $m_{i}^{(1)}$ must be strictly positive for all plant species (an axiomatic justification by the One-Life Rule will be given in the Discussion section). The same requisite applies to all herbivore and carnivore species as well.

We believe that the resource assimilation kinetics, the biomass conversion law, the two types of competition capture the essential features for plant growth. The model is used for repeatedly numerical runs with the parameter values drawn randomly for each run from uniform distributions of respective ranges. Notice that in addition to the range parameter values $a_{0}^{(k)}, c_{0}^{(k)}$ there is a trophic-connectivity probability $q_{0}^{(k)}$ and an interspecific connectivity probability $p_{0}^{(k)}$. For example, a pair of plant $i$ and $j$ has a probability of $p_{i j}^{(1)}$ to connect from $j$ to $i$ with an interference intensity $c_{i j}^{(1)}$ uniformly distributed in $\left[0, c_{0}^{(1)}\right]$. Similarly, the probability for plant $i$ to be the food source of herbivore $j$ is $q_{i j}^{(2)}$ with an encounter rate $a_{i j}^{(2)}$ uniformly distributed in $\left[0, a_{0}^{(2)}\right]$. So for $q_{0}^{(2)}<1$, a fraction of $1-q_{0}^{(2)}$ many herbivores will not be connected to a given plant. Fig. (1a) gives a qualitatively accurate depiction of our food web model for which a species is not always connected to every other species.

A fundamental difference between plants and herbivores is reflected by their resource uptake forms, the former is the time-parallel Holling Type II form and the latter is the time- 


$$
\begin{aligned}
& \left\{\begin{aligned}
\frac{d R}{d t} & =r-w \cdot * R-\left(\operatorname{diag}(R) * U^{(1)}\right) * P \\
\frac{d P}{d t} & =P . *\left(\left(\min \left(\operatorname{diag}(R) *\left(b^{(1)} * U^{(1)}\right),[], 1\right)\right)^{\prime}-d^{(1)}-m^{(1)} * P-c^{(1)} * P-U^{(2)} * H\right) \\
\frac{d H}{d t} & =H \cdot *\left(\left(b^{(2)} * U^{(2)}\right)^{\prime} * P-d^{(2)}-m^{(2)} * H-c^{(2)} * H-U^{(3)} * C\right) \\
\frac{d C}{d t} & =C *\left(\left(b^{(3)} * U^{(3)}\right)^{\prime} * H-d^{(3)}-m^{(3)} * C-c^{(3)} * C\right)
\end{aligned}\right. \\
& U^{(1)}=a^{(1)} \cdot /\left(1+\operatorname{diag}(R) *\left(h^{(1)} * a^{(1)}\right)\right) \\
& U^{(2)}=a^{(2)} * \operatorname{diag}\left(1 . /\left(1+P^{\prime} *\left(h^{(2)} * a^{(2)}\right)\right)\right) \\
& U^{(3)}=a^{(3)} * \operatorname{diag}\left(1 . /\left(1+H^{\prime} *\left(h^{(3)} * a^{(3)}\right)\right)\right)
\end{aligned}
$$

serial Holling Type II form (Murdoch 1973, Lawton 1974) which obeys the time budgeting rule for herbivorous grazing: time spend on grazing plant A cannot be spend on grazing plant $\mathrm{B}$ and so on. Another essential difference lies in the birth rates for which the former is of the law of minimum while the latter is of the law of cumulative with varying birth-to-consumption ratios $b_{j i}^{(2)}$. The same justification for using the time-parallel Holling Type II form applies to the carnivore-herbivore predation. As with the plants, interspecific competition for the animal trophics needs not to be all present but intraspecific competition must for all species.

It is obvious from the construction that higher trophics can be added to the basic model, such as to model marine ecosystems where multiple predatory chains are expected. It will be clear from our simulations of the basic three trophic model that all results can be qualitatively extrapolated to such multi-trophic foodwebs.
All simulations are done on Matlab. In Matlab syntax the right hand side of Eq. (1) is coded in Eq. 2. There instead of differentiation, $A^{\prime}$ is the transpose of a vector or matrix $A$.

Again for each simulation run, all parameter values are drown respectively from a uniformly distributed, nonnegative random number with range parameters given in Table 2. We note that all range parameter values are dimensionless and the first set is only in orders of magnitude. For example, the per-capita death rate range $d_{0}^{(2)}$ for herbivore is near zero comparing to that for carnivore because we can reasonably assume that herbivores rarely die from old age. As for another example, the handling times in range increase in order of magnitude with trophics. The same rationales went into the choices for the intraspecific and interspecific competition parameters. Also, because of the choice in the birth-to-consumption ratios with $b_{0}^{(k)} \leq 1$, all species can be thought measured in biomass with a common exchange

Table 2. Parameter Range Values

\begin{tabular}{|c|c|c|c|c|c|c|c|c|c|c|c|}
\hline \multicolumn{3}{|c|}{ Resource } & \multicolumn{3}{c|}{ Plant } & \multicolumn{3}{c|}{ Herbivore } & \multicolumn{2}{c|}{ Carnivore } \\
\hline \hline$n_{0}$ & 4 & 4 & $n_{1}$ & $100^{\dagger}$ & 100 & $n_{2}$ & 100 & 200 & $n_{3}$ & 50 & 80 \\
\hline$r_{0}$ & $10^{4 \dagger}$ & $10^{3 \dagger}$ & & & & & & & \\
\hline$w_{0}$ & 0.1 & 0.1 & & & & & & & & \\
\hline & & & $a_{0}^{(1)}$ & 0.1 & 0.1 & $a_{0}^{(2)}$ & 0.01 & 0.1 & $a_{0}^{(3)}$ & 0.1 & 0.3 \\
\hline & & & $h_{0}^{(1)}$ & $10^{-5}$ & $10^{-5}$ & $h_{0}^{(2)}$ & $10^{-3}$ & $10^{-4}$ & $h_{0}^{(3)}$ & 0.01 & $10^{-3}$ \\
\hline & & & $b_{0}^{(1)}$ & 1.0 & 1.0 & $b_{0}^{(2)}$ & 0.01 & 0.1 & $b_{0}^{(3)}$ & 0.1 & 0.1 \\
\hline & & & $d_{0}^{(1)}$ & 0.1 & 0.2 & $d_{0}^{(2)}$ & $10^{-5}$ & $10^{-5}$ & $d_{0}^{(3)}$ & 0.1 & 0.01 \\
\hline & & & $m_{0}^{(1)}$ & 0.1 & 5.0 & $m_{0}^{(2)}$ & $1^{\dagger}$ & 10 & $m_{0}^{(3)}$ & 10 & 15 \\
\hline
\end{tabular}


currency in one essential chemical element, say carbon or nitrogen for dry weight. The carnivore conversion ratio $b_{0}^{(3)}$ seems a bit too high comparing to the herbivore ratio, we will leave it as is because new simulations can be easily done for a different value and the qualitative conclusions we will reach below do not depend on this particular choice of $b_{0}^{(3)}$, nor on other range parameter values. Unless specified otherwise, parameter range values are taken from Table 2 .

Each simulation run lasts 250 units in dimensionless time for which only the last 25 time units are retained for statistical analysis. Also all species start with an initial biomass $10^{-4}$, which is also the threshold used to detect living species during the last 25 evolutionary time. A species with a biomass smaller than the threshold is assigned to the extinct category. Also, a relative tolerance $10^{-5}$ and an absolute tolerance $10^{-8}$ are used for the Matlab ODE solvers. Solver ode 15 s, ode 23 , ode 45 , ode 113 were tested, and all gave the same species count except for ode23, which is the least accurate of the four and whose count differs but with no greater than $20 \%$ of the total on average. Solver ode $15 \mathrm{~s}$, which is an adaptive-stepsize solver, was used for all simulations because of its faster speed. On a typical desktop computer, 3600 runs take between $8-16$ hours.

\section{RESULT}

We now describe some simulation results. Fig. (1b) was generated as follows. The program selected a set of random parameter values as prescribed by Table $\mathbf{1}$ and Table $\mathbf{2}$ and ran the simulation for each $\log \left(r_{0}\right)$ of 60 regularly spaced points from the interval $[-2,7]$. The relative value $\gamma=\operatorname{rand}\left(n_{0}, 1\right)$ remained the same for all $r_{0} \in\left[10^{-2}, 10^{7}\right]$ once it was generated for $\log \left(r_{0}\right)=-2$ and $r=r_{0} * \gamma$ were used
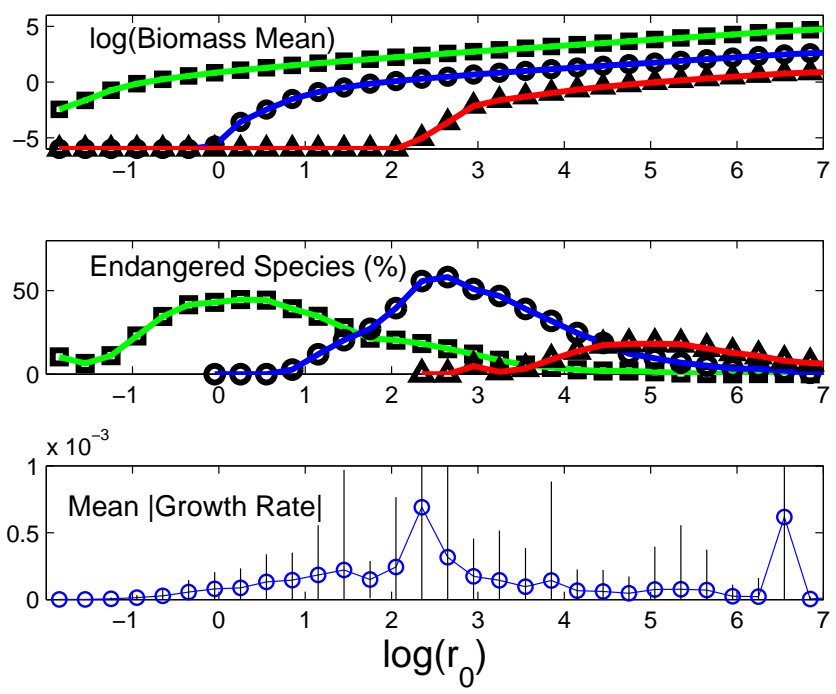

(a) for each run. Fig. (1b) shows the result of such a run. It shows that the trophication along the resource gradient: plant preceded herbivore and herbivore preceded carnivore as $r_{0}$ increases. It also shows that a species can fall or arise with resource enrichment. In Fig. (1c), we tallied up all living species (biomass $\geq 10^{-4}$ ) for each trophic level that generated Fig. (1b), and plotted two such runs. It shows that given the same range of resource abundance in $r_{0}$, particular ecosystem can behave vastly different. For example, within the range $10<r_{0}<100$, the herbivore species increases in number for one run but decreases for the other. All known empirical functional responses in species number to resource richness are shown: positive and negative correlations, sigmoidal and unimodal relationships. The only consistent correlation is between the total productivity in biomass and the resource abundance. Fig. (1d) shows the means and standard deviations of 50 runs that generated Fig. (1b). It clearly demonstrates the trophic procession to resource gradient. It also demonstrates that species richness of one trophic level is expected to decrease at the onset of trophication above it. For this particular choice in the range values of Table 2, the plant mean does not show the unimodal form like the herbivore mean does. Different choices can induce unimodalality for both plants and herbivores. Also, a particular run can exhibit the unimodalality for the carnivore number as shown in Fig. (1c). We note that whatever functional forms that show up in particular runs can be replicated for the mean curves by using the particular run's parameter values as the range values. Since the bin size is twice as wide as the partitioning interval for the $r_{0}$-interval, each plotted point is averaged over 100 data point runs.

Data on trophic biomass and population growth rate were also collected from Fig. (1c)'s runs. Fig. (2a) shows the total biomass for each trophic level. The total biomass of all
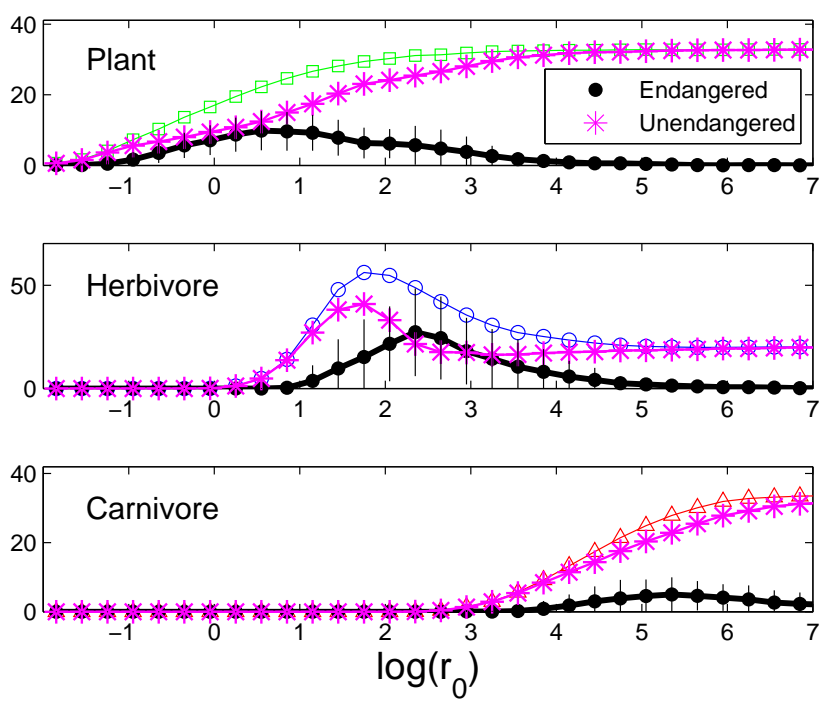

3 in (b)

Fig. (2). Statistics from the same runs as Fig. (1): (a) Top panel: plant outweighs herbivore and carnivore several orders of magnitude in biomass. Middle panel: percentages of endangered species (with negative per-capita growth rate $\left(\leq-10^{-4}\right)$ regardless biomass). Bottom panel: the mean growth rate for all living species (i.e. with dimensionless biomass $\geq-10^{-4}$ ). Stable equilibrium is expected. (b) Endangered species for each trophic level. 
species is shown in Fig. (1d) with a very modest standard deviation. More importantly, we see here that the plant is about two and four times more abundant in orders of magnitude than herbivore and carnivore respectively. (The standard deviations for the trophic biomasses are not shown since they are also modest in magnitude comparable to that of the all-trophic total from Fig. (1d)). The bottom panel of Fig. (2a) shows the growth rates $d X / d t$ in absolute value for living species only (with biomass $\geq 10^{-4}$ ). All rates are averaged over the last 25 time units of the population dynamics. It clearly shows that equilibrium steady states are expected for the foodweb.

A living species is an endangered species if its averaged per-capita rate $d X / d t / X$ over the last 25 units in time is less than $-10^{-4}$ (which is taken as zero for our ODE solver). It is a temporary designation since they happen to show up in the living species count only during the snap-shot between $225 \leq$ $t \leq 250$. Their per-capita rates may or may not become positive in future times. But if the state of the system appears to be settling down at a steady state equilibrium after a long enough time (bottom panel of Fig. (2a)) then for all practical purposes an endangered species is likely to continue to decline towards extinction. Fig. (2a) shows the percentile information for endangered species during the sampling time window. Notices that the endangered number always goes down at the appearance of a higher trophic level, meaning that endangered species are swept away sooner by predation from above than without. It also shows at the carnivorous level, resource enrichment hastens the pace of decline or extinction for endangered species because the unendangered species become more abundant and thus exert greater intratrophic pressures on endangered species. Fig. (2b) shows for each trophic level the break-up of the living species into the endangered and the unendangered categories.

Fig. (3) is generated from the same set of data as for Fig. (1) showing instead the species richness against the total biomass of the web. It was generated by dividing the biomass in the log scale into a set of 30 bins of equal size

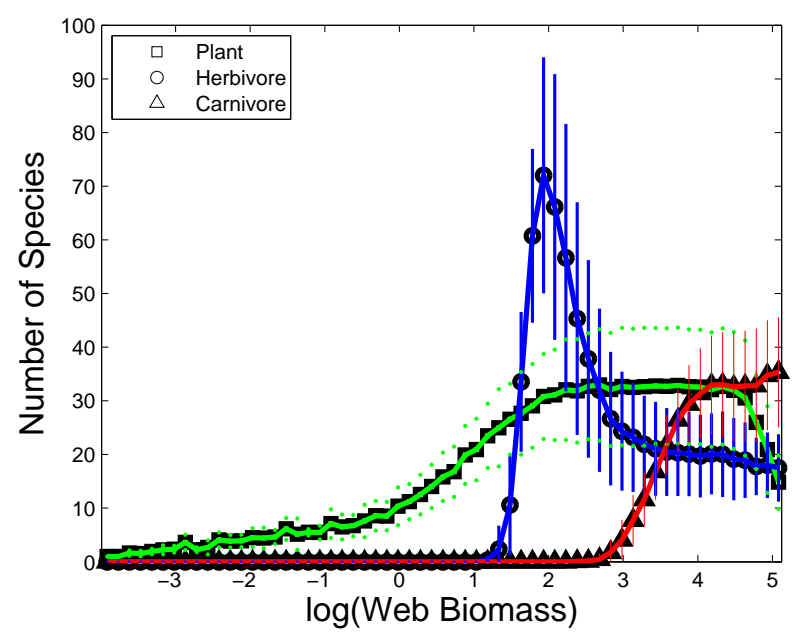

(a) and tallying up the means and standard deviations of the trophic number of species inside each bin. There is a markedly difference between Fig. (3a) and Fig. (1c) at the high end of the parameter value $r_{0}$ in that the diversityproductivity curve shows a sharp fall-off for both the plant and herbivore but a modest rise for the carnivore. Fig. (3b) was generated the same way from the data set except for fixed ranges in $r_{0}$. For example, the herbivore count from the $r_{0}$-plot Fig. (1c) in the range $10^{3}<r_{0}<10^{4}$ is strictly decreasing, yet when re-sampled against the biomass the herbivore count curve becomes unimodal. The explanation can be found in the fact that the resource richness $r_{0}$ does not give a unique response to the biomass for all arbitrary runs but rather a range of responses. Because of this property, the biomass overlaps for non-overlapping $r_{0}$ ranges, which can be seen, e.g., from the top plots of Fig. (3b). Because of such rearrangement, the species count can differ qualitatively when viewed against the resource richness and the biomass respectively. This phenomenon is the result of data sampling against different factors.

The effects of competitions are shown in Fig. (4). Fig. (4a) were generated in the same way as Fig. (1c) was except for two different sets of choice in the interspecific competition parameters. For one set, this type of competition is completely absent for both herbivorous and carnivorous species, $c_{0}^{(2)}=c_{0}^{(3)}=0$. It shows that carnivore can realize all its allowed possible species, $n_{3}=50$, when the resource is sufficiently abundant. The same also holds for the herbivore before the emergence of the carnivore, and the herbivorous species number is significantly higher than that with interspecific competition $c_{0}^{(2)}>0$ even under the predation pressure from above. In addition, these changes in the top two trophics have a small effect on the plant diversity and almost no effect on the total web biomass. For comparison, a second set of data is also shown for different values of the interspecific parameters $c_{0}^{(2)}=0.05, c_{0}^{(3)}=0.5$, for which the
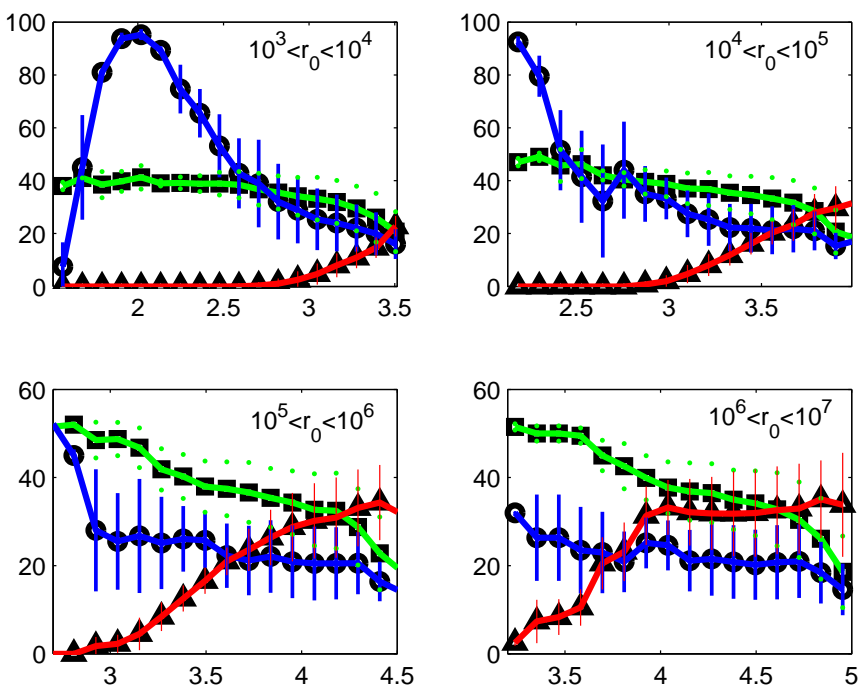

3 in (b)

Fig. (3). Statistics from the same runs as Fig. (1): (a) Re-sampling the species numbers against the total biomass of the web. (b) The same diversity-biomass sampling is carried out for four different ranges in $r_{0}$ : showing different $r_{0}$ ranges give rise to overlapped ranges in biomass. It is this property that explains the qualitative difference between, e.g., Fig. (1d) and the top-right plot for the range $10^{3}<r_{0}<10^{4}$. 


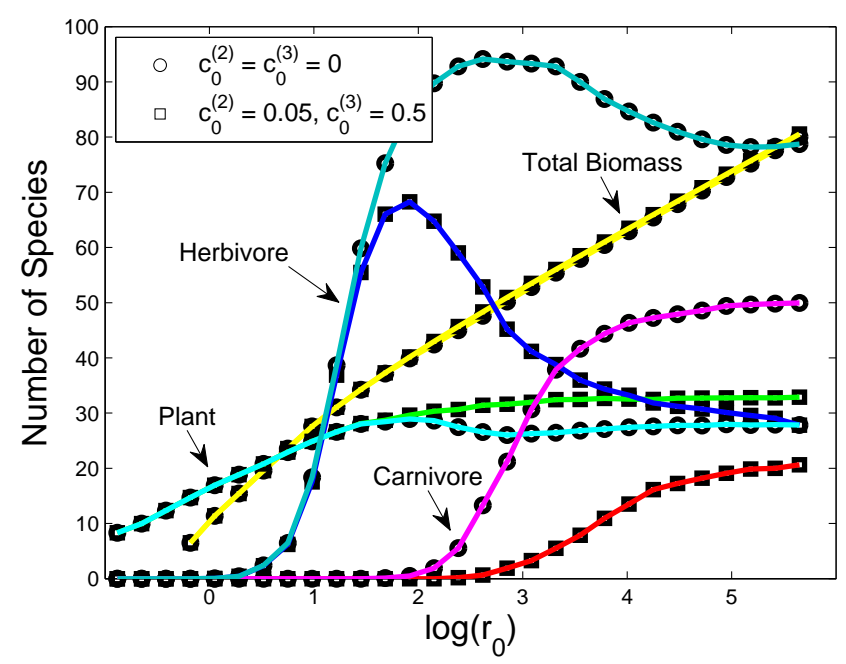

(a)

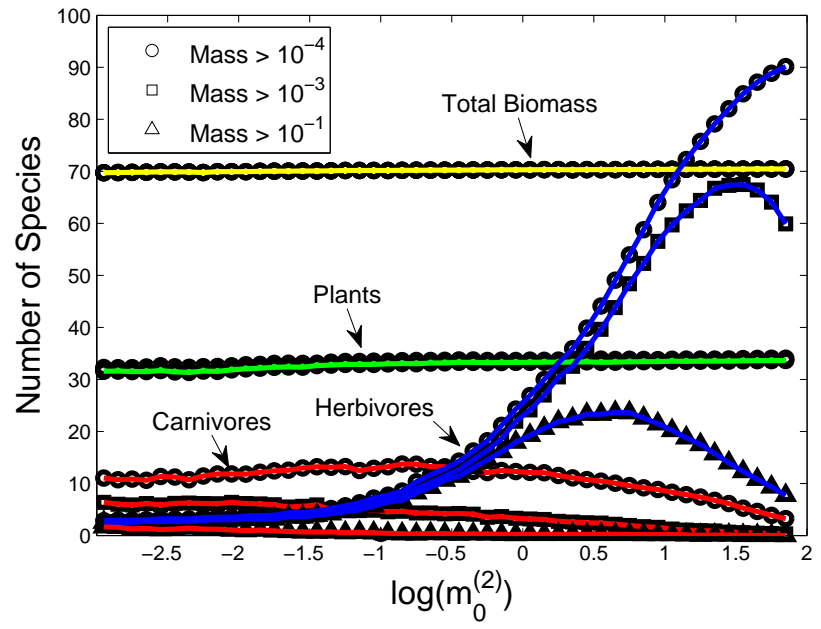

3 in (b)

Fig. (4). (a) Two different sets of over 60 runs with different interspecific competition strengths. Without interspecific competition, the carnivore mean reaches its allowed possible number $n_{3}=50$, and the herbivore mean almost reaches its allowed possible number $n_{2}=100$ before the appearance of carnivore. But the mean total biomass remains the same. (b) For the fixed $r_{0}=10^{3}$, it shows the means over 50 runs against herbivore's intraspecific parameter. Shown here are the nearly constant total biomass (in a false scale), the total plant number, but graded herbivore and carnivore numbers. Competitive exclusion occurs without intraspecific competition, i.e. $m_{0}^{(2)}=0$.

allowed possible numbers, $n_{2}=100, n_{3}=50$, are far away from living species counts for the top two trophics. There is little change in the total web biomass. That is, there are fewer but more massive species.

Fig. (4b) was generated similarly but with all range parameters fixed from Table 2 except for the intraspecific parameter for the herbivore, $m_{0}^{(2)}$, which ranges from $10^{-3}$ to $10^{2}$. It shows that there is little change in the plant diversity nor in the total biomass, which tends to be conserved. But it shows the phenomenon of competitive exclusion in the herbivorous level when intraspecific competition is absent $m_{0}^{(2)} \sim 0$. Its diversity curve is unimodal against the competition intensity $m_{0}^{(2)}$ in graded levels in biomass. Carnivorous diversity suffers little until its more massive herbivorous preys splinter into more numerous but less abundant species. In other words, unlike the interspecific competition, there is an optimal intensity for intraspecific competition with res-pect to diversity for a given biomass, either too little or too much an intensity has a negative impact on species number at that given biomass.

Fig. (5a) was generated in the same way as Fig. (1c) except that the second set of parameter values from Table $\mathbf{2}$ was used and the range was fixed at $10^{-3}$ for two resources while that for the other two is allowed to vary. This may resemble the latitudinal gradient scenario for which the water-energy increases toward the equator while other resource limitations in space and nutrients remain equal. It shows that because of the resource limitations, the total biomass as well as the species counts saturate after the range for the varying resources surpass some value beyond which they are no longer limiting. The other qualitative properties described above also hold for this set of parameter range values, such as the phenomenon of trophication, the existence of endangered species, the trophic separations in biomass, the opposite effects of the two types of competition, and the stability of the foodweb.

In addition to these properties, Fig. (5b) presents another piece of information. It was generated as follows. Let $X(t)=$ $[P(t) ; H(t) ; C(t)]$ denote the near steady state at the end of the simulation run $t \sim 250$ for which some species are considered extinct when its biomass is $\leq 10^{-4}$ and the others are considered living with greater than $10^{-4}$ in biomass. Let $\sigma=$ $\left(i_{1}, i_{2}, \ldots, i_{k}\right)$ denote the component indexes for the extinct species, i.e. $X_{\sigma} \leq 10^{-4}$ component-wise. Then the averaged per-capita rates for the non-living species $X_{\sigma}$ were evaluated, $\left(d X_{i} / d t\right) / X_{i}=F_{i}(X(t))$, at the near equilibrium state $X(t)$ for $225<t<250$. Of which we tabulated the number of those extinct species $X_{\sigma}$ whose averaged per-capita growth rates were positive (numerically $\geq 10^{-4}$ ). The set of such species are referred to as the secondary trophication pool in the plot. For all practical purposes, such a species fits the mathematical definition of being competitive which we will give in the Discussion section. As we will explain later in that section, any species from the pool can reinvade the foodweb and coexist with all or some other living species. For example, one can add at least one herbivore almost immediately at the plant trophication $r_{0} \sim 10^{-1}$. Alternatively, one can add at least one carnivore from the secondary trophication pool at the herbivore trophication $r_{0} \sim 10$. Similarly, if a living carnivore from habitats $r_{0}>100$ is also competitive in habitats $10<r_{0}<100$ where no carnivores are allowed to emerge on their own because of resource limitation on the primary trophication, then that living carnivore can invade that primary plant-herbivore habitats.

Fig. (6a) is a plot of plant diversity against two varying parameters: the resource range parameter $r_{0}$ and the number 


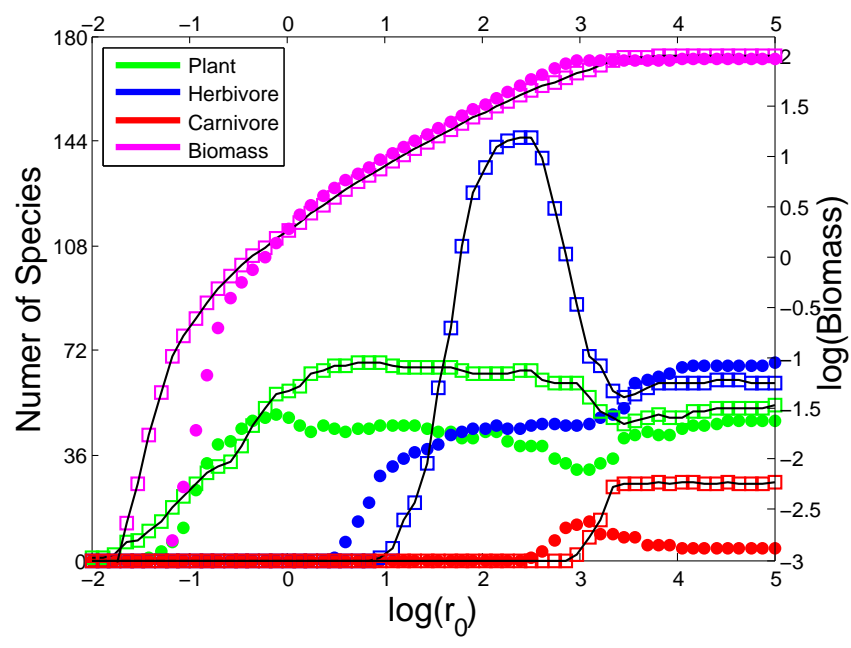

(a)
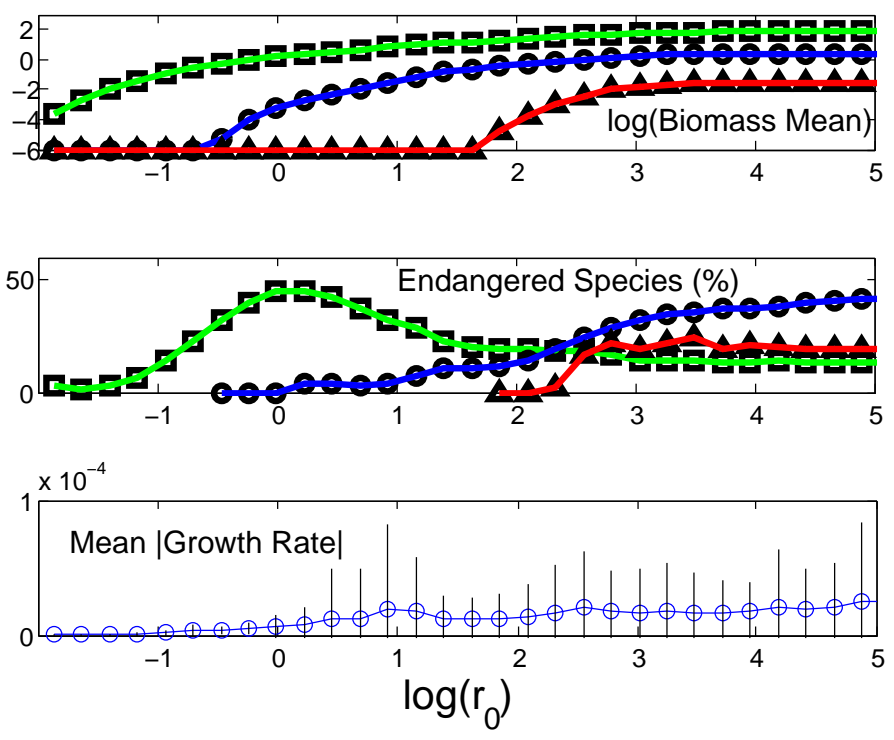

(c)

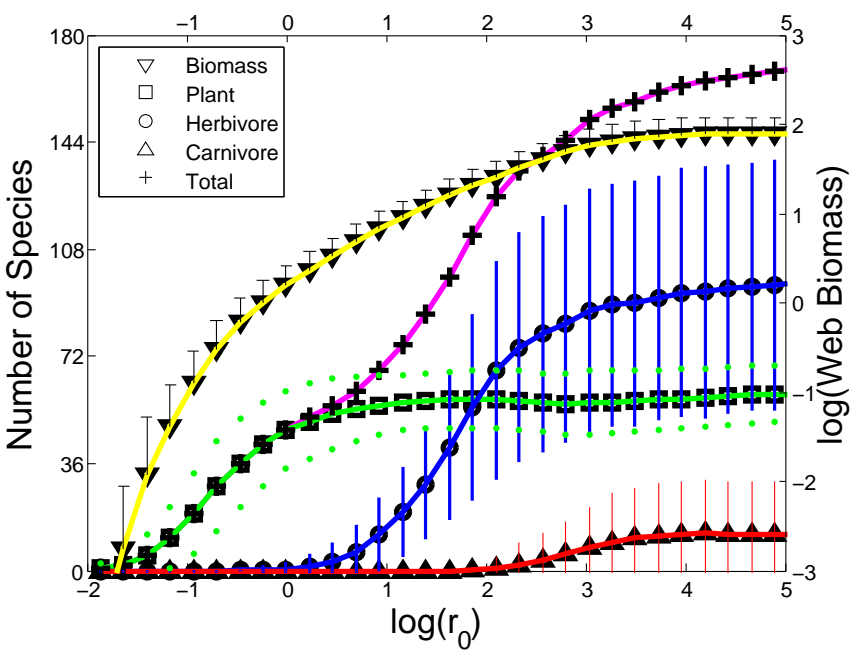

3in (b)
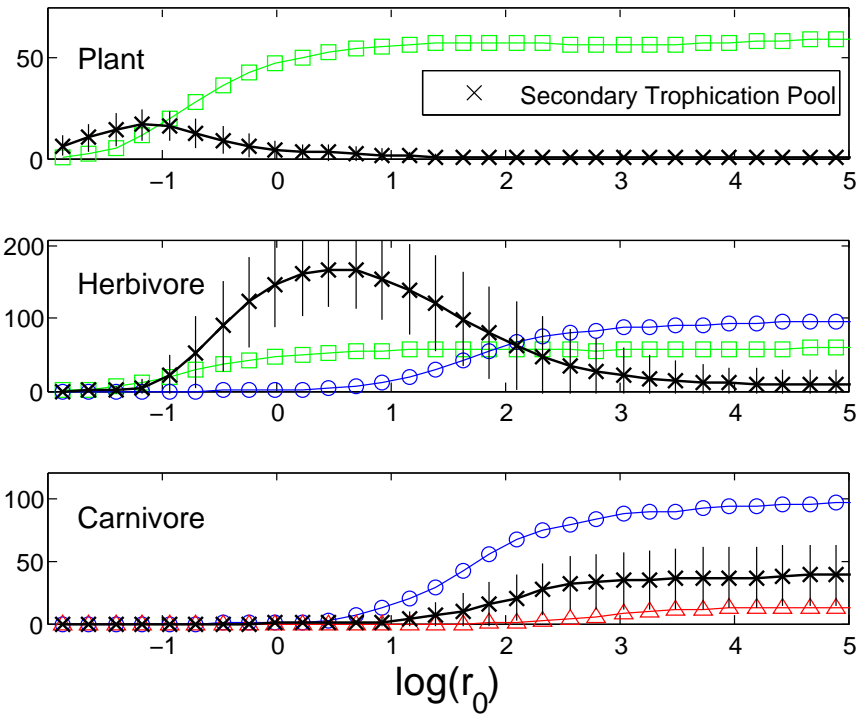

3 in $(d)$

Fig. (5). (a) Similar simulations as Figs. (1 and 2) except for the second set of parameter values from Table 2 and that the ranges for two of the resources are limited at $10^{3}$ while the other two resources are allowed to vary with the bifurcation parameter $r_{0}$. For $r_{0}$ beyond $10^{3}$, both the biomass and the species counts saturate because of the Law of Minimum on plant growth. Each data point was generated from 196 runs. The legend of plot (b) also applies to plots (c,d).

of possible plant species $n_{1}$. Except for $a_{0}^{(2)}=0$ which effectively removes the top two trophics from the system, other parameter values are the same as the first set given in Table 2. The result shows that with the presence of interspecific competition, $c_{0}^{(1)}=10^{-3}$, the plant diversity saturates along the $n_{1}$ axis as well as along the $r_{0}$ axis, i.e. at higher end of both $n_{1}$ and $r_{0}$, the number of plants remains about the same but some or all of them will become more massive in total biomass. This shows for sufficiently large $n_{k}$, all results described above are not dependent on our choice in the possible numbers of species. However, if the species pool is limited as in all field studies which are inevitably short in time, then the ecosystems functioning is expected to depend on the pool numbers and for particular systems such dependence can be either positive or negative in correlation as implied by Fig. (1b) and Fig. (5a).

\section{DISCUSSION}

As mentioned in the Method section, each simulation run can be thought to simulate speciation or primary succession of a particular and isolated habitat. The simulation setup can be interpreted for the establishment of an ecosystem when all possible species are given an equal chance with a uniformly small initial population. It is an in silico simulation of "neutral speciation". Although it is not explicitly said to model allopatric speciation by which species diverge while being isolated geographically or sympatric speciation by which species diverge while inhabiting the same place via polyploidization, hybrid formation, or sexual selection, our 


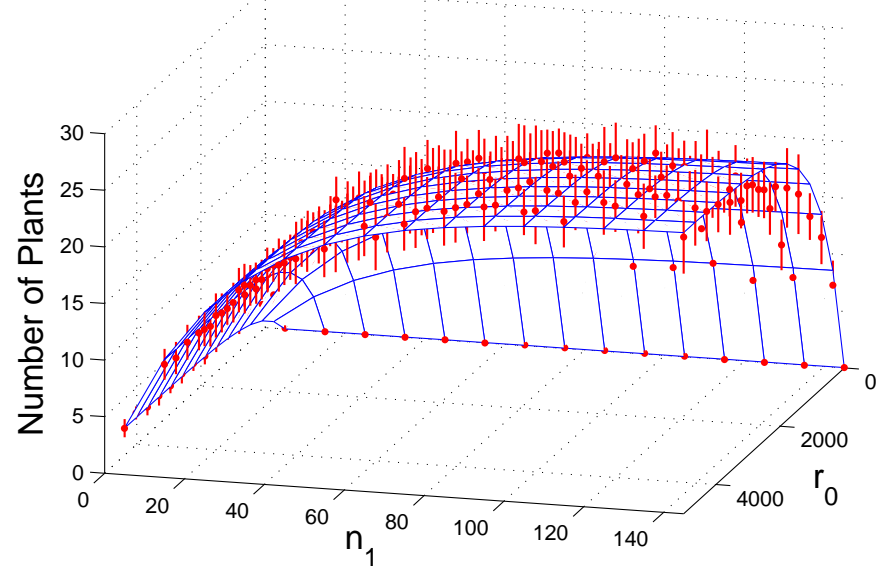

3in (a)

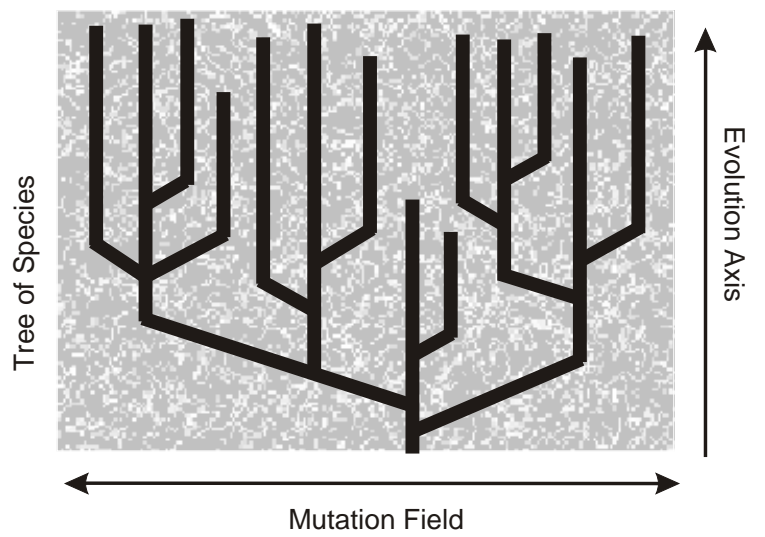

3in (b)

Fig. (6). (a) The statistical effect of the number of possible plant species $n_{1}$ on the realized (or living) plant number, each dot and bar denote the mean and standard deviation over 100 runs without herbivores (i.e. $a_{0}^{(2)}=0$ ), showing selection by competition allows only a bounded number of plants to exist no matter how large the possible number $n_{1}$ is. Similar conclusions apply to other trophic species. (b) A conceptual summary of the model and its result for which the grainy background represents all possible species allowed by random mutations referred to as the mutation field.

"neutral speciation" does not rule them out. For example, it is absolutely conceivable that the parameter values of one simulation run are so similar to another simulation run that it permits the interpretation of one species going through either an allopatric or sympatric speciation. Specifically, such divergences can be thought to start with some small deviations in parts of one species' genome from another in a given habitat. Drawing parameter values randomly in the way our simulation did is a general way to model the effect rather than the mechanism of such speciations, and to model the latter their mathematical formulations must be in place first which is lacking at this point. Nevertheless, since our design is focused on the statistics of an ensemble of many individual runs, it allows us to have something to say about the number of surviving species aggregated over many habitats contemporaneously or over many temporal episodes of speciation and succession of one habitat. One obvious finding is that individual systems can behave very differently and thus sweeping principles cannot be obtained from them (Figs. (1c) and (5a)). Another unambiguous finding is the phenomenon of trophication that higher trophic levels appear only at higher resource gradient. As another general rule, trophic species are separated in orders of magnitude in biomass, which is consistent with the observation that in biomass plant dominates herbivore and herbivore dominates carnivore. Some less obvious aspects of our result are commented below.

\section{One-Life Rule vs. Paradoxes}

Based on what we know about life on Earth we can axiomatically assume that every organism has only one finite life. To translate this postulate into mathematics, let $x_{t}$ be the population of one species in individual count or total biomass, then the per-capita growth in any time span $\tau>0$ must satisfy this fundamental constraint,

$$
\left(x_{t+\tau}-x_{t}\right) / x_{t}=x_{t+\tau} / x_{t}-1 \geq-1 .
$$

If we assume because of resource limitation in space and nutrient necessary for life that

$$
\lim _{t \rightarrow \infty} \frac{x_{t+\tau}}{x_{t}}=0
$$

for any $\tau>0$, then the expression below summaries what is referred to as the One-Life Rule (Deng 2008, Deng 2009),

$$
\lim _{x_{t} \rightarrow \infty} \frac{x_{t+\tau}-x_{t}}{x_{t}}=-1^{+},
$$

where the superscript "+" means the limit converges always from above to -1 . This rule is not obeyed by almost all discrete population models (Deng 2008, Deng 2009) nor by those discrete models for biodiversity (Hughes 2000, Ives 2002, McPeek 2008). This is one of the two main reasons we used a continuous rather than a discrete model for this paper. (The other reason to reject discrete models in ecological modeling is because of the fact that they in general violate the Time Invariance Principle which is the basis for the reproducibility of independent experiments (Deng 2008, Deng 2009)).

For continuous population models, the RosenzweigMacArthur producer-consumer model,

$$
\left\{\begin{array}{l}
\frac{d x}{d t}=r x\left(1-\frac{x}{K}\right)-\frac{a x y}{1+h a x} \\
\frac{d y}{d t}=b \frac{a x y}{1+h a x}-d y
\end{array}\right.
$$

violates the One-Life Rule as well. Specifically, it is the predator's population that does it. The argument goes as follows. Suppose the equations model a predator-prey system and suppose as a thought experiment that the prey population is maintained by an experimenter at a constant level. Then the model would predict that the predator grow at an exponential rate with 


$$
y(t)=y_{0} e^{\gamma t},
$$

which violates the One-Life Rule since,

$$
\lim _{y(t) \rightarrow \infty}(y(\tau+t)-y(t)) / y(t)=e^{\gamma \tau}-1>-1
$$

regardless the sign of $\gamma=b a x /(1+h a x)-d$, meaning an infinite many predators can be maintained over any time interval of length $\tau>0$. Similarly, all multi-trophic extensions of the Rosenzweig-MacArthur model (Lehman 2000, Shurin 2001, Mouquet 2002, Thebault 2005, Gallet 2007, Gross 2007, Goudard 2008) suffer the same flaw. When such models are used to extract global properties at a long time scale, paradoxical predictions are inevitable. In fact, all paradoxes in theoretical ecology are the artifacts of models violating the One-Life Rule. They include the Enrichment Paradox (Rosenzweig 1971, Deng 2007a, Deng 2008), the Competi-tion Exclusion Principle or the Plankton Paradox (Hutchison 1961, MacArthur 1964, Armstrong 1980, Deng 2006), and the Biological Control Paradox (Luck 1990, Deng 2007a), none of which is supported by consensual empirical findings.

On the other hand, the logistic model $d x / d t=x(r-m x)$ with intraspecific competition $m>0$ obeys the One-Life Rule (Deng 2008). The particular linear form, $m x$, is just one of the simplest ways to model intraspecific competition. For example, assume the Holling Type II predation form with predator self-interference (Beddington 1975, De Angelis 1975b) but without the additional linear intraspecific competition term, a predator's population can be modeled by $d y / d t=y(\operatorname{bax} /(1+h a x+m y)-d)$. With the prey density $x$ fixed at any constant supply, one can explicitly show that the predator has a carrying capacity and satisfies the One-Life Rule. As a standing conjecture, any species whose equation has a carrying capacity when all other interacting species are fixed at constant densities obeys the One-Life Rule. The single most important consequence from the One-Life Rule lies in the fact that all aforementioned pathological paradoxes disappear from theoretical ecology (Deng 2006, Deng 2007a, Deng 2008). For example, contrary to a conventional wisdom (Rosenzweig 1971), our result supports the paradigm that enrichment plus the One-Life Rule lead to stability and coexistence.

\section{Large but Stable}

In fact, this result has been proved mathematically for the Lotka-Volterra model in Ahmad (1996), Montes de Oca (1995). To state a simpler version of the result of Ahmad (1996), Montes de Oca (1995), let,

$$
d N_{i} / d t=N_{i}\left(b_{i}-m_{i} N_{i}-\sum_{j=1, j \neq i}^{n} c_{i j} N_{j}\right)
$$

for $1 \leq i \leq n$ be the Lotka-Volterra model for $n$ competing species. Then all species will coexist at a globally stable equilibrium state if this condition holds,

$$
b_{i}>\sum_{j=1, j \neq i}^{n} c_{i j} \frac{b_{j}}{m_{j}}
$$

for all $1 \leq i \leq n$, where $b_{j} / m_{j}$ is the carrying capacity of species $j$ in the absence of interspecific competition $c_{i j}=0$ for all $i, j$. One can see from this condition that if all the subsystem carrying capacities are bounded from above, then efficiency with large $b_{i}$ or weak interspecific competition with small $c_{i j}$ guarantees equilibrium coexistence. Stating the condition equivalently,

$$
\sum_{j=1, j \neq i}^{n} \frac{b_{j}}{b_{i}} \frac{c_{i j}}{m_{j}}<1,
$$

we see that if the efficiency parameters $b_{i}$ are comparable with each other, then either weak interspecific competition (small $c_{i j}$ ) or strong intraspecific competition (large $m_{i}$ ) or both guarantees a stable coexistence. Our simulations are consistent with these theoretical results. So the stability of a foodweb obeying the One-Life Rule (nonzero $m_{i}>0$ ) is not the result of being sufficiently complex as suggested by Elton (Elton 1958), nor being significantly small as suggested by May (May 1972). It usually happens for organisms of finite life span in sufficiently rich habitat.

Unlike other models with paradoxes, the complexityinstability theory of May is not linked to the violation of One-Life Rule. Instead May's theory does not apply to the Lotka-Volterra model nor to our foodweb model Eq. (1). In order for his theory to apply, the linearization, $d X / d t=A X$, of the system at the coexisting equilibrium point in question must allow itself to be simultaneously scaled so that the diagonal entries are all equal to -1 , i.e. $A=B-I$ with $b_{i j}=0$ for $i$. This requirement cannot be met even for the two species system (3) with or without intraspecific competition as one of the diagonal entry becomes zero at a Hopf bifurcation point (Kuznetsov 1995). As shown recently by Allesina (2008), which is consistent with the stability result of Ahmad (1996) and Montes de Oca (1995), large systems can be stable if May's unrealistic condition is abandoned for ecological models.

\section{Limit Cycle and Chaos --- An Exception}

Not all ecosystems are at steady equilibriums, some are locked in limit cycles, or even possibly in chaotic oscillations. However, such oscillatory systems tend to be few in comparison and situated at high latitudes with greater limitations in resources (Elton 1942, Odum 1971, Krebs 1974, Finerty 1980, Calder 1983, Peterson 1984, Hanski 1993, Ellner 1995, Framstad 1997). In other words, population oscillations represent an exception rather than the rule in the literature (Odum 1971, Ellner 1995). Food chain models of three or more species consistently demonstrate the possibility of chaotic dynamics (Hogeweg 1978, Gilpin 1979, Waltman 1983, Hastings 1991, Smith 1994, Bockelman 2004, Bockelman 2005, Deng 2006, Deng 2007b). However, all these models assume Holling's functional forms for the predators for which their handling time of prey is a measure of their predating efficiency or a lack of it. It is not known that the Lotka-Volterra model, which assumes zero handling time for all its species, is capable of chaos generation. This curious state of research points to a possible explanation that efficiency suppresses complexity. This can be seen from Fig. (2a) that (transient) cycles are possible for particular systems with growth rates further away from zero, but with reduced 
handling times for efficiency (Table 2), the systems tend to become more stable as shown in Fig. (5c). There is another way other than having small handling times for stabilization. It was demonstrated in Deng (2006) that for models compliant with the One-Life Rule, all chaos can be eliminated by resource enrichment or by reproductive efficiency or by both even with significant handling times of all species. This implies that limit cycles or chaos in population may only be found in habitats of poor resources or with inefficient species, consistent with the empirical findings from the literature (Elton 1942, Krebs 1974, Finerty 1980, Calder 1983, Peterson 1984, Hanski 1993, Framstad 1997). This result is consistent with our simulations as efficient species tend to out-compete inefficient ones when both are randomly generated at comparable odds and followed up for a long time. For those rare cyclic systems we observe today, they may happen to be in their evolutionary transient towards a steady equilibrium or in a temporary deviation from such a state. Our current experimental design is not set up to capture such oscillatory transients, but their existence in our model is not in doubt.

\section{Mathematical Definition of Competitive Species}

Often left unsaid mathematical modeling in ecology to a large extent is to translate Darwin's theory of evolution to mathematics. However, the central concept of competitiveness of a species has not been explicitly defined mathematically in the literature, but alluded to in MacArthur (1967). A species $Y$ is said to be competitive in a system of species $X_{1}, X_{2}, \ldots, X_{n}$ (with $Y \neq X_{i}$ for any $i$ ) if along any stable steady state of the $X$-system the time-averaged percapita growth rate $\frac{1}{Y} \frac{d Y}{d t}$ for species $Y$ is positive. For example, if the stable steady state is an equilibrium, $X(t) \equiv \bar{X}$, then $Y$ is competitive with respect to the $X$ system provided that the per-capita rate for $Y$ satisfies $F(X, 0)$ $>0$ where $\frac{d Y}{d t}=Y F(X, Y)$. Similarly, if the steady state is a limit cycle, $X(t+T)=X(t)$ with $T$ being the period, then the $Y$-competitive condition is $\frac{1}{T} \int_{0}^{T} F(X(t), 0) d t>0$. If the $X$ steady state is a chaotic attractor and $X(t)$ is a dense orbit on the attractor, then the $Y$-competitive condition is $\liminf _{T \rightarrow \infty} \frac{1}{T} \int_{0}^{T} F(X(t), 0) d t>0$. This definition is perfectly consistent with the definition of local stability of attractors of dynamical systems as demonstrated in MacArthur (1967). A theorem can be stated as follows: If $a$ species $Y$ is competitive in an $X$-system which has a global attractor $\mathrm{A}$, then the extended steady state $(\mathrm{A}, 0)$ in the extended $X Y$-system is unstable, and if there exists a global attractor for the $X Y$-system then species $Y$ must be positive in biomass along the new XY-attractor. This result implies a stable foodweb can only be invaded by a competitive species. Note that this result leaves it open the question whether or not $Y$ is competitive in any subsystem of the $X$ system and in general it is not. This means the timing of speciation of $Y$ is important --- it can invade a system only when the system is ready for it, i.e. when it becomes competitive. In general, the new system will reorganize itself so that some species may be driven to extinction by the invader $Y$, but being just another typical system with randomly fixed parameters, the new system is expected to converge to a new equilibrium state. This is the theoretical basis for Fig. (5d).

\section{Endangered Species with Drifting Equilibrium}

As shown by our simulations, a typical ecosystem is expected to settle down at a stable equilibrium, and as a result over any given time interval it is expected to be in the transient to that equilibrium. However, due to exogenous and endogenous stochasticities, the asymptotic equilibrium state constantly changes itself. In other words, any deviation is expected only to re-aim the system to a new stable equilibrium, and the time trajectory of the system treks along a quasi-equilibrium state we call it the drifting equilibrium. Since the growth rate of a species at an equilibrium point is zero, the rate at the drifting equilibrium is expected to bounce around zero. So just looking at the growth rate of a species at a single point in time is insufficient to conclude its endangered status. However, if its growth rate is negative when averaged over an extended period of time, then for practical purposes it can be classified as endangered, the criterion used for our simulations. If furthermore its population in head count is also small, then it can be classified as threatened with extinction. Our simulations show that endangered species is always expected at every drifting equilibrium state. We can certainly cross reference of endangered species with their biomass which our simulation setup is capable of as shown in Fig. (4b), but our model is not constructed to translate the total biomass of a species into its population head count because the physiology of the species (in terms of biomass per individual) is not built into the model. This is the main reason that only the criterion of endangered species is defined and used in this paper. We certainly expect that a significant proportion of those endangered is also threatened with extinction.

According to the latest update of the IUCN Red List, there are 17,291 species out of the 47,677 assessed species are threatened with extinction. Almost all are assessed by their diminishing numbers over a long monitoring period and by their changing habitats at a rate faster than what we think natural adaptation can cope. Human's activities certainly contribute to the current wave of extinction, but they do not explain past extinctions before the origin of man. Our result shows part of the extinction takes place naturally as ecosystems progress along their drifting equilibriums. Endangered species have been a constant presence throughout time, and extinction happens even under what we would think to be the "best" circumstance when the resources going upward along its gradient (Figs. (1) and (5a)).

\section{Effects with Other Range Parameters}

We have simulated the effects of $r_{0}, c_{0}^{(k)}, m_{0}^{(k)}, n_{k}$. The range of $b_{0}^{(k)}$ is automatically bounded from above by 1 because of the law of mass conservation. The effect for the remaining range parameters is secondary, and most can be 
inferred from what we have learned from the simulations presented above. For example, increasing $d_{0}^{(k)}$ decreases the competitiveness of the $k$ th-trophic species, and therefore decreases the trophic's species richness. Increasing $a_{0}^{(k)}$ or decreasing $h_{0}^{(k)}$ makes the $k$ th-trophic species more competitive, increasing the trophic's species count but decreases the $(k-1)$ st-trophic species count, or the endangered count in particular. As for the connectivity parameters, decreasing the interspecific connectivity probability $p_{0}^{(k)}$ has the same effect as decreasing the interspecific competitive strength $c_{0}^{(k)}$, thus increasing the corresponding trophic's species number. Similarly reasoning can be applied to the intertrophic connectivity $q_{0}^{(k)}$.

\section{Future Direction: Succession and Asymptotic Trophica- tion}

With respect to Fig. (5d), the simulation shows that not all possible species can establish themselves by starting small, but some species (i.e. the secondary trophication pool) can speciate or become competitive only after a primary drifting equilibrium has been established. In other words, this simulation gives a theoretical basis for succession and colonization by emigration, both can affect the speciesrichness. For example, any one of the species from the three secondary trophication pools of Fig. (5b) can be added to the drifting equilibrium to alter the trophication against the resource gradient. That is, the trophication of herbivore can be advanced from $r_{0} \sim 10$ to a smaller $r_{0}$ value at $r_{0}=10^{-1}$ either through secondary speciation or through invasion of herbivores from the primary trophication of herbivore at the richer habitat $r_{0}>10$. This process of colonization and succession by emigration will alter the diversity-resource curve, and a future project is to simulate this iterative process to see if the diversity-resource curve will eventually settle down with all niches occupied by competitive species and to see if the connectance for the asymptotic species counts is consistent with empirical findings (Melián 2004). The preliminary result on competitive species (Fig. 5d) reconciles our finding on trophication along the resource gradient and the fact that carnivores, such as the polar bear, are found at high latitudes where they were not part of the primary trophication.

\section{Future Direction: Fitting Model to Data}

Our model seems to fit qualitatively well with known facts: that the center of both allopatric and sympatric speciation is within a zone of the equator where water and light are in the greatest abundance; the biomass separation between plants and its consumers; the sequential trophication of species; and the stability of geographically isolated ecosystems. Our model also allows quantitative manipulation of species numbers at all trophic level with simple changes in the intensity of intraspecific and interspecific competitions. These features should allow us to fit our model not only to the behaviors of a particular system but also to the aggregated properties of many particular systems. However, we have a case of theory outpacing practice here.
The key obstacle to fitting our model to data is the incompleteness of the latter. For example, by the latest count of IUCN (IUCN 2008) there is about known 1.6 million species of which there are about 300,000 plants, 120,000 fungi (Hawkins 2001), and 230,000 marine species (Crist 2009), totaled from different habitats which for practical purposes can be considered geographically isolated for species census. We can easily calibrate the model to fit these numbers if the number of isolated geographies is also given. But such a fit is of limited use because the actual species number is estimated at least 10 time higher (Wilson 2000). For a particular system, one also encounters the same missing-data problem. For example, there is no problem to fit our model to the PET relationship against the species numbers in birds, small mammals, and amphibians from Currie (1991). But the missing information on the plant species number and the number of habitats would render such a fit incomplete even to the 0th-order. That is, without the missing information, there will be many completely free parameters any possible values of which will give the same single-dimensional fit. The challenge is to determine which parameters are theoretically impossible to determine and which parameters can be fixed from such incomplete fit. Understanding such data limitations is important in order to realize the model's predictive potential by successively removing the limitations one at a time.

\section{Modeling Neutrality and Neo-Darwinism}

The modern theory of evolution by natural selection is predicated on two mechanisms, the source of selection by genetic mutations and the fixation of mutations by survival of the fittest. Regarding genetic mutations, Kimura's neutral theory (Kimura 1983) is hard to dismiss. The finding that genetic mutation is not geographically dependent (Currie 2004, Bridle 2009, William 2009) further strengthens the neutrality idea used here for our model for biodiversity, which in turn implies chance plays a predominate role in speciation. In other words, at the molecular level speciation is largely undirected and mostly random. The set of all possible mutations for different species forms what is referred to as the mutation field. The randomly picked pool of possible species for our model (with $n_{1}$ plants, $n_{2}$ herbivores, and $n_{3}$ carnivores) is a representation of this neutral mutation field. The change along the resource gradient in $r_{0}$ can be thought to mimic the environmental changes when life first appeared on Earth. Our simulation for the number of living species along the resource gradient in $r_{0}$ can be thought to model the speciation and trophication at that beginning. In particular, it predicts that species from progressively higher trophic levels originated from progressively richer habitats in liquid water and solar energy. More importantly, because of the fundamental constraint by the One-Life Rule, all species evolve along a drifting equilibrium. An argument perhaps can be made that because of the stability of the drifting equilibrium most genetic mutations are neutral. In other words, drifting equilibrium at the phynotypic level perhaps is the basis for the genetic drift at the molecular level. Fig. (6b) gives a conceptual representation of our model as well as a graphical summary of the model, showing that the tree of species percolates through the mutation field along a drifting equilibrium, consisting of 
paths of least resistance in terms of natural selection for competitive species.

We conclude the paper with a comment on human's impact on biodiversity that is implied by our result and hard to ignore. First an observation, the rise of the human species can be considered as the trophication of one super species atop all foodwebs. This reality alone will accelerate the demise of most endangered species (Fig. 2b). It seems to be another reasonable observation that our transforming natural habitats into agricultural land at the rate and scale parallel the human population explosion is the primary component of our species's competitive edge against other species, and the increasingly fragmented and shrinking natural habitats intensify the interspecific as well as the intraspecific competitive intensities for other species. As suggested by our result (Fig. 4a) the increase in interspecific competition will inevitably lead to biodiversity loss. Furthermore, the increase in intraspecific competition of other species will drive more massive species to extinction (Fig. 4b). Although according to our simulation these massive species will be replaced by more but less massive species, this is possible only if the total biomass of all non-human species can be maintained, which is not likely without the necessary space for plants to grow and for animals to roam. The combined effect is a planet of fewer species --- a commentary hardly new but here borne out by a mathematical model.

\section{ACKNOWLEDGEMENT}

Special thanks to Dr. Sabrina Russo for serendipitously introducing the author to the subject of biodiversity while working on the joint RUTE program of the Department of Mathematics and the School of Biological Sciences at UNL.

\section{REFERENCES}

Ahmad, S \& Lazer, AC (1996) One species extinction in an autonomous competition model. In: Proceedings of The First World Congress on Nonlinear Analysis '92, Vol. I. Walter de Gruyter, Berlin 35968.

Allesina, S \& Pascual, M (2008) Network structure, predator-prey modules, and stability in large food webs. Theoretical Ecology, 1, 55-64.

Armstrong, RA \& McGehee, R (1980) Competive exclusion. American Naturalist, 115, 151-70.

Beddington, JR (1975) Mutual interference between parasites or predators and its effect on searching efficiency. Journal of Animal Ecology, 44, 331-40.

Bockelman, B, Deng, B, Green, E, Hines, G, Lippitt, L \& Sherman, J (2004) Chaotic coexistence in a top-predator mediated competitive exclusive web. Journal of Dynamics and Differential Equations, $16,1062-92$

Bockelman, B, Deng, B (2005) Food web chaos without subchain oscillators. Internatial Journal of Bifurcation and Chaos, 15, 348192.

Bradley, JC, Hillebrand, H, Harpole, WS, Gross, K \& Ptacnik, R (2009) Separating the influence of resource "availability" from resource "imbalance" on productivity-diversity relationships. Ecology Letters, 12, 475-87.

Bridle, JR, Gavaz, S \& Kennington, WJ (2009) Testing limits to adaptation along altitudinal gradients in rainforest Drosophila. Proceedings of Royal Society B: Biological Sciences, 276, 1507-15.

Briggs, JC (1995) Global Biogeography, Developments in Palaeontology and Stratigraphy, Elsevier: Amsterdam Vol 14.

Broham, L \& Cardillo, M (2003) Testing the link between the latitudinal gradient in species richness and rates of molecular evolution. Journal of Evolutionary Biology, 16, 200-7.
Calder III, WA (1983) An allometric approach to population cycles of mammals. Journal of Theoretical Biology, 100, 275-82.

Clark, JS, Dietze, M, Chakraborty, S, Agarwal, PK, Ibanez, I, LaDeau, S \& Wolosin, M (2007) Resolving the biodiversity paradox. Ecology Letters, 10, 647-62.

Crist, DT, Scowcroft, G \& Harding Jr., JM (2009) World Ocean Census. Firefly Books: Toronto.

Currie, DJ (1991) Energy and large-scale patterns of animal and plantspecies richness. American Naturalist, 137, 27-49.

Currie, DJ \& Francis, AP (2004) Regional versus Climatic Effect on Taxon Richness in Angiosperms: Reply to Qian and Ricklefs. American Naturalist, 163, 780-5.

Darlington, PJ (1957) Zoogeography: The Geographical Distribution of Animals. Wiley, New York.

Darwin, C (1859) On the origin of species by means of natural selection. John Murray, London.

De Angelis, DL (1975a) Stability and connectance in food web models. Ecology, 56, 238-43.

De Angelis, DL, Goldstein, RA \& O'Neill, RV (1975b) A model for trophic interaction. Ecology, 56, 881-92.

Deng, B (2006) Equilibriumizing all food chain chaos through reproductive efficiency. Chaos, 16, 043125. DOI: 10.1063/1.2405711

Deng, B, Jessie, S, Ledder, G, Rand, A \& Srodulski, S (2007a) Biological control does not imply paradox. Mathematical Biosciences, 208 26-32.

Deng, B \& Loladze, I (2007b) Competitive coexistence in stoichiometry chaos. Chaos, 17, 033108. DOI: 10.1063/1.2752491

Deng, B (2008) The Time Invariance Principle, the absence of ecological chaos, and a fundamental pitfall of discrete modeling. Ecological Modelling, 215, 287-92.

Deng, B (2009) Can discrete modelers work without the TIP? Ecological Modelling, 220, 2600-01.

Dijkstra, FA, Hobbie, SE, Reich, PB \& Knops, JMH (2005) Divergent effects of elevated $\mathrm{CO} 2, \mathrm{~N}$ fertilization, and plant diversity on soil $\mathrm{C}$ and $\mathrm{N}$ dynamics in a grassland field experiment. Plant Soil, 272, 41-52.

Doak, DF, Bigger, D, Harding, EK, Marvier, MA, O'Malley, RE \& Thomson, D (1998) The Statistical inevitability of stabilitydiversity relationships in community ecology. Amercan Naturalist, 151, 264-76.

Ellner, S \& Turchin, P (1995) Chaos in a noisy world: new methods and evidence from time-series analysis. Amercan Naturalist, 145, 34375 .

Elton, CS \& Nicholson, M (1942) The ten-year cycle in numbers of the lynx in Canada. Journal of Animal Ecology, 11, 215-44.

Elton, CS (1958) The Ecology of Invasions by Animals and Plants. Methuen and Co: London, UK.

Finerty, JP (1980) The population ecology of cycles in small mammals. Yale University Press: New Haven.

Framstad, E, Stenseth, NC, Bjornstad, ON \& Falck, W (1997) Limit cycles in Norwegian lemmings: Tensions between phase-dependence and density-dependence. Proceedings of the Royal Society B: Biological Sciences, 264, 31-38.

Freedman, HI \& So, JWH (1985) Global stability and persistence of simple food chains. Mathematical Biosciences, 76, 69-86.

Gallet, R, Alizon, S, Comte, P-A, Gutierrez, A, Depaulis, F, van Baalen, M, Michel, E \& Müller-Graf, CDM (2007) Predation and disturbance interact to shape prey species diversity. American Naturalist, 170, 143-54.

Gilpin, ME (1979) Spiral chaos in a predator-prey model. American Naturalist, 113, 306-8.

Goudard, A \& Loreau, M (2008) Nontrophic interactions, biodiversity, and ecosystem functioning: an interaction web model. American Naturalist, 171, 91-106.

Gross, K \& Cardinale, BJ (2007) Does species richness drive community production or vice versa? Reconciling historical and contemporary paradigms in competitive communities. American Naturalist, 170, 207-20.

Hanski, I, Turchin, P, Korpimaki, E, \& Henttonen, H (1993) Population oscillations of boreal rodents: regulation by mustelid predators leads to chaos. Nature, 364, 232-5.

Hastings, A \& Powell, T (1991) Chaos in a three-species food chain. Ecology, 72, 896-903. 
Hogeweg, P \& Hesper, B (1978) Interactive instruction on population interactions. Computers in Biology and Medicine, 8, 319-27.

Hawkins, BA (2001) Ecology's oldest pattern? Trends in Ecology and Evolution, 16, 470.

Hawksworth, DL (2001) The magnitude of fungal diversity: the 1.5 million species estimate revisited. Mycological Research, 105, 1422-32.

Hillebrand, $H$ (2003) On the generality of the latitudinal diversity gradient. Computers in Biology and Medicine, 163, 192-211.

Holling, CS (1959) Some characteristics of simple types of predation and parasitism. Canadian Entomologist, 91, 385-98.

Hubbell, SP (2001) The Unified Neutral Theory of Biodiversity and Biogeography. Princeton University Press: Princeton, NJ.

Hughes, JB \& Roughgarden, J (2000) Species diversity and biomass stability. American Naturalist, 155, 618-27.

Huston, MA, Aarssen, LW, Austin, MP, Cade, BS, Fridley, JD, Garnier, E, Grime, JP, Hodgson, J, Lauenroth, WK, Thompson, K, Vandermeer, JH \& Wardle, DA (2000) No consistent effect of plant diversity on productivity. Science, $289,1255 \mathrm{a}$.

Hutchison, GE (1961) The paradox of the plankton. American Naturalist, $95,137-45$.

IUCN, Wildlife in a Changing World: An analysis of the 2008 Red List of Threatened Species, http://iucn.org/about/work/programmes/ species/red list/review/

Ives, AR \& Hughes, JB (2002) General relationships between species diversity and stability in competitive systems. American Naturalist, $159,388-95$

Kimura, M (1983) The Neutral Theory of Molecular Evolution. Cambridge University Press: Cambridge.

King, AW \& Pimm, SL (1983) Complexity, diversity, and stability: A reconciliation of theoretical and empirical results. American Naturalist, 122, 229-39.

Kinzig, AP \& Pacala, S (2001) Successional biodiversity and ecosystem functioning. In: Kinzig, AP, Tilman, D \& Pacala, S (Eds) The functional consequences of biodiversity : empirical progress and theoretical extensions. Princeton University Press: Princeton 175212.

Krebs, CJ \& Myers, JH (1974) Polulation cycles in small mammals. Advances in Ecological Research, 8, 267-399.

Kuznetsov, YuA (1995) Elements of Applied Bifurcation Theory. SpringerVerlag: New York.

Lawton, JH, Beddington, JR \& Bonser, R (1974) Switching in invertebrate predators. In: Usher, MB \& Williamson, MH (Eds) Ecological Stability. Chapman and Hall: London 141-58.

Lehman, CL \& Tilman, D (2000) Biodiversity, stability, and productivity in competitive communities. American Naturalist, 156, 534-52.

Lotka, AJ (1925) Elements of Physical Biology. Williams and Wilkins: Baltimore, Md.

Luck, RF (1990) Evaluation of natural enemies for biological control: a behavior approach. Trends in Ecology and Evolution, 5, 196-9.

MacArthur, RH \& Levins, R (1964) Competiton, habitat selection, and character displacement in a patchy environment. Proceedings of the National Academy of Sciences, 51, 1207-10.

MacArthur, RH \& Levins, R (1967) The limiting similarity, convergence, and divergence of coexisting species. American Naturalist, 101, 377-85.

MacArthur, RH \& Wilson, EO (1963) An equilibrium theory of insular zoogeography. Evolution, 17, 373-87.

May, RM (1972) Will a large complex system be stable? Nature, 238, 4134.

May, RM (1973) Stability and Complexity in Model Ecosystem. Princeton University Press: Princeton.

May, RM (2009) Food-web assembly and collapse: mathematical models and implications for conservation. Philosophical Transactions of the Royal Society B: Biological Sciences, 364, 1643-6.

McPeek, MA (2008) The ecological dynamics of clade diversification and community assembly. American Naturalist, 172, E270-E284.

McGrady-Steed, J, Harris, PM \& Morin, PJ (1997) Biodiversity regulates ecosystem predictability. Nature, 390, 162-5.

McNaughton, SJ (1994) Biodiversity and function of grazing ecosystems. In: Schulze, E-D \& Mooney, HA (Eds) Biodiversity and Ecosystem Function. Springer-Verlag: New York 361-84.

Melián, CJ \& Bascompte, J (2004) Food web cohesion. Ecology, 85, 352-8.
Michaelis, L \& Menten, MI (1913) Die Kinetik der Invertinwirkung. Biochemische Zeitschrift, 49, 333-69.

Michalski, J \& Arditi, R (1999) The complexity-stability problem in food web theory. What can we learn from exploratory models? In: Blasco, F \& Weill, A (Eds) Advances in Environmental and Ecological Modelling. Elsevier, Paris 91-119.

Monod, J \& Jacob, F (1961) General conclusions: teleonomic mechanisms in cellular metabolism, growth and differentiation. Cold Spring Harbor Symposia on Quantitative Biology, 26, 389-401.

Montes de Oca, F \& Zeeman, ML (1995) Balancing survival and extinction in nonautonomous competitive Lotka-Volterra systems. Journal of Mathematical Analysis and Applications, 192, 360-70.

Mouquet, N \& Loreau, M (2002) Coexistence in metacommunities: the regional similarity hypothesis. American Naturalist, 159, 420-6.

Murdoch, WW (1973) The functional response of predators. Journal of Applied Ecology, 10, 335-42.

O'Brien, EM (1998) Water-energy dynamics, climate and prediction of woody plant species richness: an interim general model. Journal of Biogeography, 25, 379-98.

Odum, EP (1971) Fundamentals of Ecology. W.B. Saunders Company: Philadelphia.

Peterson, RO, Page, RE \& Dodge, KM (1984) Wolves, moose, and the allometry of population cycles. Science, 224, 1350-2.

Pfisterer, AB \& Schmid, B (2002) Diversity-dependent production can decrease the stability of ecosystem functioning. Nature, 416, 84-6.

Pimm, SL (1984) The complexity and stability of ecosystems. Nature, 307, 321-6.

Qian, H, Fridley, JD \& Palmer, MW (2007) The latitudinal gradient of species-area relationships for vascular plants of North America. American Naturalist, 170, 690-701.

Rosenzweig, ML (1971) Paradox of enrichment: destabilization of exploitation ecosystems in ecological time. Science, 171, 385-7.

Rosenzweig, ML (1995) Species Diversity in Space and Time. Cambridge Univ. Press: Cambridge, UK.

Rosenzweig, ML \& MacArthur, RH (1963) Graphical representation and stability conditions of predator-prey interactions. American Naturalist, 97, 209-23.

Rozdilsky, ID \& Stone, L (2001) Complexity can enhance stability in competitive systems. Ecology Letters, 4, 397-400.

Scheiner, SM \& Willig, MR (2005) Developing unified theories in ecology as exemplified with diversity gradients. American Naturalist, 166, 458-69.

Schulze, E-D \& Gerstberger, P (1994) Functional aspects of landscape diverstiy: a Bavarian example. In: Schulze, E-D \& Mooney, HA (Eds) Biodiversity and Ecosystem Function. Springer-Verlag: New York 453-66.

Shurin, JB \& Allen, EG (2001) Effects of competition, predation, and dispersal on species richness at local and regional scales. American Naturalist, 158, 624-37.

Smith, HL \& Waltman, P (1994) The theory of the Chemostat---Dynamics of Microbial Competition. Cambridge Studies in Mathematical Biology, Cambridge University Press: Cambridge.

Stanley, SM (1984) Marine mass extinction: a dominant role for temperature. In: Nitecki, MH (Ed) Extinctions. University of Chicago Press: Chigago 69-117.

Thebault, E \&Loreau, M (2005) Trophic interactions and the relationship between species diversity and ecosystem stability. American Naturalist, 166, E95-E114.

Tilman, D (1988) Plant Strategies and the Dynamics and Structure of Plant Communities. Princeton University Press: Princeton.

Tilman, D, Reich, PB \& Knops, JMH (2006) Biodiversity and ecosystem stability in a decade-long grassland experiment. Nature, 441, 62932 .

Volterra, V (1926) Fluactuations in the abundance of species, considered mathematically. Nature, 118, 558-60.

Waide, RB, Willig, MR, Steiner, CF, Mittelbach, G, Gough, L, Dodson, SI, Juday, P \& Parmenter, R (1999) The relationship between productivity and species richness. Annual Review of Ecology and Systematics, 30, 257-300.

Wallace, AR (1876) The geographical distribution of animals, 2 Vols. Macmillan and Co: London, UK.

Wallace, AR (1878) Tropical nature and other essays. Macmillan: London, UK. 
Waltman, P (1983) Competition Models in Population Biology. SIAM: Philadelphia.

Whittaker, RH (1967) Gradient analysis of vegetation. Biological Reviews, 42, 207-64.

William, EK, Vergeer, P, Kenta, T, Davey, MP, Burke, T, Woodward, FI, Quick, P, Mannarelli, M-E, Watson-Haigh, NS \& Butlin, R (2009)
Variation at range margins across multiple spatial scales: environmental temperature, population genetics and metabolomic phenotype. Proceedings of the Royal Society B: Biological Sciences, 276, 1495-506.

Wilson, EO (2000) A global biodiversity map. Science, 289, 2279.

Received: November 25, 2009

Revised: March 19, 2010

Accepted: March 27, 2010

(C) Bo Deng; Licensee Bentham Open.

This is an open access article licensed under the terms of the Creative Commons Attribution Non-Commercial License (http://creativecommons.org/licenses/by$\mathrm{nc} / 3.0 /$ ), which permits unrestricted, non-commercial use, distribution \& reproduction in any medium, provided the work is properly cited. 